\title{
Prdm6 controls heart development by regulating neural crest cell differentiation and migration
}

Lingjuan Hong, ${ }^{1} \mathrm{Na} \mathrm{Li},{ }^{1}$ Victor Gasque, ${ }^{1}$ Sameet Mehta, ${ }^{2}$ Lupeng $\mathrm{Ye},{ }^{3}$ Yinyu $\mathrm{Wu},{ }^{3}$ Jinyu Li, ${ }^{1}$ Andreas Gewies, ${ }^{4}$ Jürgen Ruland, ${ }^{5}$ Karen K. Hirschi, ${ }^{1,6}$ Anne Eichmann, ${ }^{1,7}$ Caroline Hendry, ${ }^{3}$ David van Dijk, ${ }^{1,8}$ and Arya Mani ${ }^{1,3}$

${ }^{1}$ Cardiovascular Research Center, Department of Internal Medicine, ${ }^{2}$ Yale Center for Cenome Analysis, and ${ }^{3}$ Department of Genetics, Yale University School of Medicine, New Haven, Connecticut, USA. ${ }^{4} \mathrm{Helmholtz}$ Zentrum München, Munich, Germany. ${ }^{5}$ The Technical University of Munich, Munich, Germany. ${ }^{6}$ University of Virginia School of Medicine, Charlottesville, Virginia, USA. 'Department of Cellular and Molecular Physiology, Yale University School of Medicine, New Haven, Connecticut, USA. ${ }^{8}$ Yale Department of Computer Science, Yale University, New Haven, Connecticut, USA.

The molecular mechanisms that drive the acquisition of distinct neural crest cell (NCC) fates is still poorly understood. Here, we identified Prdm6 as an epigenetic modifier that temporally and spatially regulates the expression of NCC specifiers and determines the fate of a subset of migrating cardiac NCCs (CNCCs). Using transcriptomic analysis and genetic and fate mapping approaches in transgenic mice, we showed that disruption of Prdm6 was associated with impaired CNCC differentiation, delamination, and migration and led to patent ductus arteriosus (DA) and ventricular noncompaction. Bulk and single-cell RNA-Seq analyses of the DA and CNCCs identified Prdm6 as a regulator of a network of CNCC specification genes, including Wnt1, Tfap2b, and Sox9. Loss of Prdm6 in CNCCs diminished its expression in the pre-epithelial-mesenchymal transition (pre-EMT) cluster, resulting in the retention of NCCs in the dorsal neural tube. This defect was associated with diminished $\mathrm{H} 4 \mathrm{~K} 20$ monomethylation and G1-S progression and augmented Wnt1 transcript levels in pre-EMT and neural tube clusters, which we showed was the major driver of the impaired CNCC migration. Altogether, these findings revealed Prdm6 as a key regulator of CNCC differentiation and migration and identified Prdm6 and its regulated network as potential targets for the treatment of congenital heart diseases.

Conflict of interest: The authors have declared that no conflict of interest exists.

Copyright: (c) 2022, Hong et al. This is an open access article published under the terms of the Creative Commons Attribution 4.0 International License.

Submitted: October 21, 2021

Accepted: January 13, 2022

Published: February 2, 2022

Reference information: /CI Insight. 2022;7(4):e156046

https://doi.org/10.1172/jci.

insight.156046.

\section{Introduction}

Neural crest cells (NCCs) are a transient population of multipotent cells unique to vertebrates that arise from neural folds during embryonic development (1) and migrate throughout the body, giving rise to diverse cell lineages. In mice, cardiac NCCs (CNCCs) - precursors to the heart - are generated at 8-8.5 dpc and arrive in the distal outflow at E9-9.5 (2). Targeted ablation of Wnt1-expressing CNCCs at 8.5 dpc in mice results in a complex phenotype of craniofacial and cardiovascular outflow tract defects (3). Similarly, the impaired function of CNCCs in humans underlies the pathogenesis of various complex human congenital disorders collectively known as cardio craniofacial syndromes (4). Cell lineage analyses have helped uncover the spatial and temporal diversification of NCC lineages $(5,6)$. However, the mechanisms underlying the pleiotropic effects of CNCCs are not understood owing to a lack of insight into how subsets of CNCCs are specified and how their subsequent fate is determined.

PRDM6 is a smooth muscle cell-specific (SMC-specific) histone methyltransferase and a member of the PRDM family of transcriptional repressors. It is expressed in the cardiac outflow tract and the ductus arteriosus (DA), a small artery that connects the aorta and pulmonary artery (7). Mice globally deficient for Prdm6 are embryonically lethal because of abnormal vascular patterning (8). In humans, loss-of-function mutations in the PRDM6 gene have been the first, and to this date, the only known genetic cause of the familial nonsyndromic patent DA (PDA) (9). PDA is an extreme example of an isolated cardiac disease that results from failure of closure of the DA, a derivative of the sixth pharyngeal arch, which is largely derived from premigratory CNCCs (10). The tunica media of the DA and other pharyngeal arch-remodeled arteries 
consists mainly of neural crest-derived SMCs (11). Syndromic PDA is caused by mutation in the NCC-specifier TFAP2B $(12,13)$. However, whether a defect in NCCs underpins the nonsyndromic PDAs and whether $P R D M 6$ plays a causal role in this process have never been investigated. Here, we combined bulk and single-cell RNA-Seq (scRNA-Seq) with 2 mouse models and identified Prdm6 as a hub that regulates a network of genes involved in CNCC specification and orchestrates the delamination, migration, and differentiation of a subgroup of CNCCs required for DA formation in a Wnt1-dependent manner.

\section{Results}

Prdm6 expression in CNCCs is required for embryonic heart development. NCCs contribute to the development of the sixth aortic arch and its derivatives, the DA and pulmonary artery, and give rise to the tunica media of these arteries (14). To determine whether Prdm6 is involved in the specification of NCCs and formation of the DA, we crossbred Prdm6 ${ }^{l / f l}$ and Wnt1-Cre2 mice for several generations to generate Prdm6 $6^{A l f l}$ Wnt1Cre2 mice. According to an earlier study, Wnt1 expression in CNCCs starts on dpc 8-8.5 (15), while Prdm6 expression has been shown to be readily detectable in the embryo by E9.5 using Northern blot analysis (8). No gross syndromic abnormalities were noted in Prdm6 $6^{\text {flfl }}$ Wnt1-Cre2 mice (Supplemental Figure 1A; supplemental material available online with this article; https://doi.org/10.1172/jci.insight.156046DS1). Prdm6 ${ }^{l / f l}$ Wnt1-Cre2 mice died on P0.5 with widely open DAs (Figure 1A). There was no abnormality of the great arteries or their branching (Figure 1A). The cross-section examination of DAs in Prdm6 ${ }^{\text {flfl }}$ Wnt1-Cre2 mice showed a large and widely patent lumen (Figure 1B). The examination of the skeleton of the Prdm6 $6^{\text {fl }}$ fl Wnt1-Cre2 mice showed only a small defect in the frontal bone at the junction of the anterior fontanel (Figure 1C) and no other defects (Figure 1D). The examination of the heart showed biventricular noncompaction but no other structural defect (Figure 1E). These findings indicate that Prdm6 expression in CNCCs is required for proper closure of the DA and the development of compacted myocardium during embryonic development. The cardiovascular traits were completely penetrant in both male and female mice (Table 1)

Loss of Prdm6 diminishes the contribution of CNCCs to the myocardium and DA. To determine how CNCC fate is affected by loss of Prdm6, we performed lineage tracing of Wnt1-CNCCs using Wnt1-Cre2 ZsGreen1 mice $\left(\mathrm{Prdm}^{+/+}\right)$and Prdm6 ${ }^{\text {Ilfl }}$ Wnt1-Cre2 ZsGreen1 mice in which Prdm6 expression was disrupted specifically in CNCCs. In both cases, all Wnt1-CNCCs and their progeny were labeled with ZsGreen1 cell body (Figure 2A). The transverse sections of the cardiac neural crest in Prdm6 ${ }^{l / f l}$ Wnt1-Cre2 ZsGreen1 embryos at E9.5 showed significant retention of ZsGreen1-positive cells in the neural tube (red arrows) and increased cell number in the neural tube compared with $\mathrm{Prdm}^{+/+}$Wnt1-Cre2 ZsGreen1 (WT) mice, suggestive of impaired NCC delamination (Figure 2, B-D). Examination of the great vessels and semilunar valves at P0.5 revealed no significant difference between Prdm $6^{\text {flfl }}$ Wnt1-Cre2 ZsGreen1 mice and WT controls (Supplemental Figure 1B). However, the cross-sectioning of the heart at E9.5 showed reduced ZsGreen1-positive cells and thinning of the myocardium in Prdm6 $6^{\sharp l f l}$ Wnt1-Cre2 ZsGreen1 mice compared with WT mice (Figure 2, E-G).

It has been shown that the SMCs in the tunica media of the DA are derived from CNCCs (16). The examination of the DA of Prdm6 $6^{A / f l}$ Wnt1-Cre2 ZsGreen1 mice at E17.5 showed dramatically reduced ZsGreen1-positive (NCC-derived) cells in the tunica media compared with those of the WT littermates (Figure 3, A and B). In contrast to controls, the DA wall in Prdm6 $6^{\text {Ilfl }}$ Wnt1-Cre2 ZsGreen1 mice was thinner, and only a fraction of its SMCs was NCC-derived (Figure 3B). In addition, the NCC-derived SMCs of Prdm6 $6^{A / f}$ Wnt1-Cre2 ZsGreen1 mice had an irregular shape (Figure 3C) outlined by straight yellow lines and had infiltrated and disrupted the endothelial layer, stained with CD31 antibody and outlined by dotted yellow lines (Figure 3C). Endothelial cells of the DA are not NCC derived and undergo apoptosis at the onset of ductus closure (17), as shown in the WT ductus (Supplemental Figure 2A). In contrast, the endothelial layer of the patent ductus in Prdm6 $6^{\text {flfl }}$ Wnt1-Cre2 ZsGreen1 mice appeared intact at birth. Unexpectedly, we noted the costaining of ZsGreen1 and VE-cadherin in several cells within the ductus endothelial layers (Supplemental Figure 2, A and B) and fewer NCC-derived SMCs (Supplemental Figure 2C) in Prdm6 ${ }^{\text {Ilfl }}$ Wnt1-Cre2 ZsGreen1 mice versus WT littermates. These findings indicated the critical role of Prdm6 in NCC differentiation.

We then examined whether impaired proliferation of NCCs contributes to the failure of DA closure. We examined cell proliferation at E17.5 in Prdm6 ${ }^{\text {flfl }}$ Wnt1-Cre2 ZsGreen1 mice and their WT littermates using Ki67 staining (Figure 3D). We observed no significant difference in the percentage of Ki67-positive NCC-derived or non-NCC-derived SMCs between the 2 genotypes (Figure 3E). At birth, the DA cells are no longer proliferative and undergo apoptosis $(18,19)$. Accordingly, the Ki67 staining of the ductus in the WT mice showed absence of ductal cell proliferation (Supplemental Figure 2D). In contrast, ZsGreen1-negative and 
A

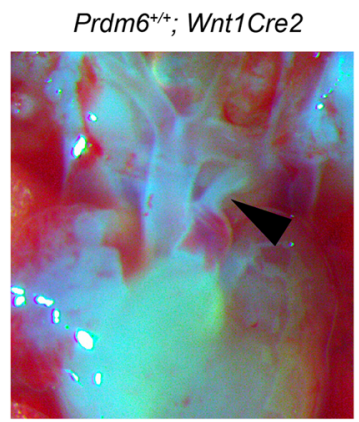

C
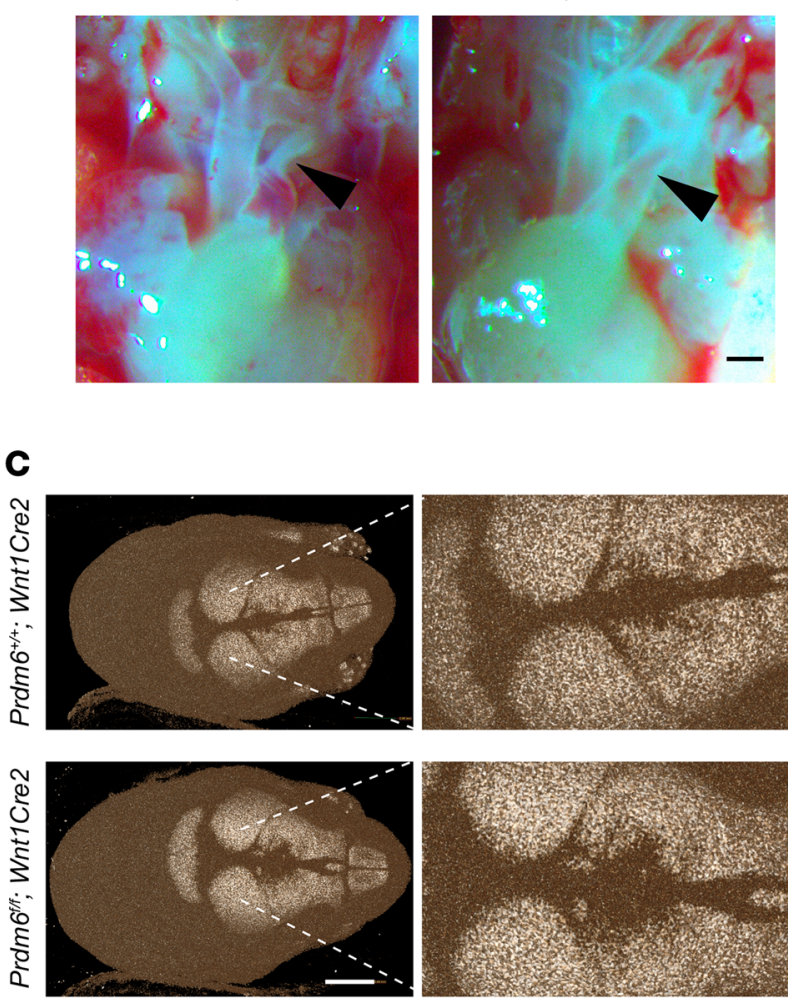

E

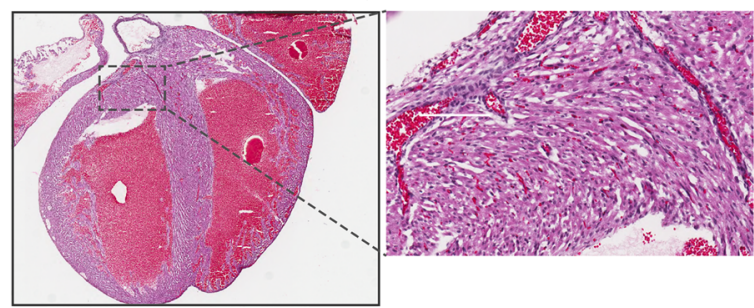

B

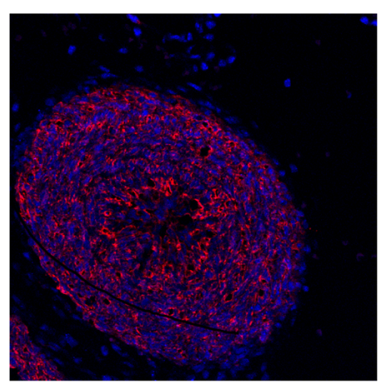

D
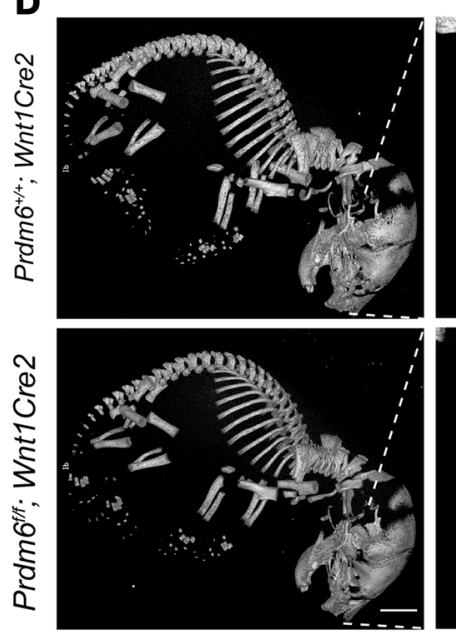

Prdm6 6 $;$ Wnt1Cre2

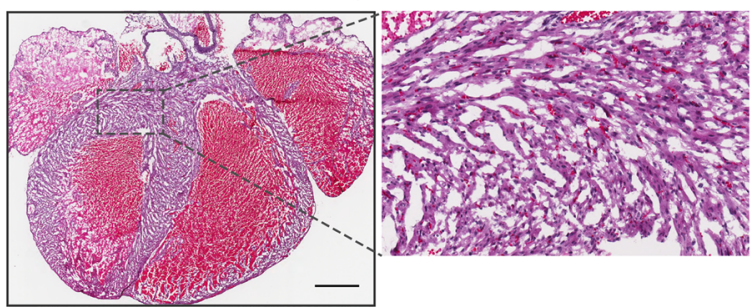

Prdm6 fff; Wnt1Cre2

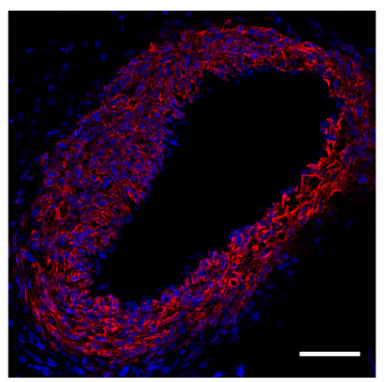

DAPI/aSMA
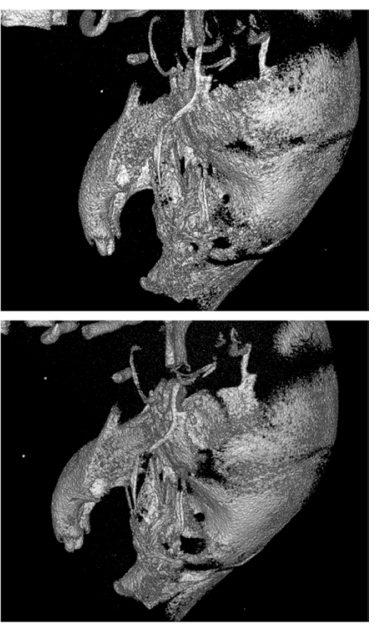

Figure 1. NCC-specific Prdm6-KO mice are born with PDA and noncompacted myocardium. (A) The gross appearance of a control heart (left) and Prdm6 $6^{f / f l}$ Wnt1-Cre2 heart (right) and great cardiac vessels at P0.5. The closed DA and patent DA are shown by black arrowheads. Scale bar: $200 \mu \mathrm{m}$. (B) The confocal images of representative cross-sections of the DA of control (left) and Prdm6 f/ff Wnt1-Cre2 (right) pups at P0.5 stained with antibodies against $\alpha$ SMA (red) and DAPI (blue). Scale bar: $100 \mu \mathrm{m}$. (C) The computer micrographs of control (top) and Prdmf flf Wnt7-Cre2 (bottom) pups' skulls at P0.5, demonstrating small defect in the frontal bone at the junction of the anterior fontanel from apical views. Scale bar: $2 \mathrm{~mm}$. The right panels show the same images magnified for better visualization. (D) The computer micrographs of control (top) and Prdm6 ff/fl Wnt1-Cre2 (bottom) pups' skeletons and skulls at P0.5 from lateral views. Scale bar: $3 \mathrm{~mm}$. (E) H\&E staining of control (left) and Prdm6 f/f Wnt1Cre2 (right) pup hearts, demonstrating biventricular noncompaction but no other gross structural anomalies. Scale bar: $500 \mu \mathrm{m}$. All controls were the corresponding littermates ( $n=3-6$ for each group).

to a lesser degree ZsGreen1-positive ductal SMCs in Prdm6 $6^{\text {I/f }}$ Wnt1-Cre2 ZsGreen1 mice were Ki67 positive (Supplemental Figure 2E). Taken together, these findings indicated that the patency of the DA in Prdm6 ${ }^{l / f}$ Wnt1-Cre2 ZsGreen1 mice was not caused by impaired proliferation of NCC-derived SMCs. In addition, increased proliferation of non-NCC-derived SMCs appeared as insufficient to compensate for reduced NCC-derived SMCs in Prdm6 $6^{n / f}$ Wnt1-Cre2 ZsGreen 1 mice. This finding is consistent with a prior report that NCC-derived and not mesodermal-derived SMCs are responsible for ductal closure (20).

In addition, numerous ZsGreen1-positive cells were detected in the ventricular septum and to a lesser degree in the innermost layer of the left and right ventricles of WT pups at P0.5. The number of ZsGreen1-positive cells in the myocardium of Prdm6 ${ }^{\text {H/fl }}$ Wnt1-Cre2 ZsGreen1 mice was also significantly diminished compared with WT mice (Supplemental Figure 2, F and G), a finding that correlated with the ventricular noncompaction in these mice and suggested impaired NCC migration to the heart. Together, these results suggested that loss of Prdm6 expression resulted in impaired NCC migration and 
Table 1. Genotype and phenotype of PO.5 pups

\begin{tabular}{|c|c|c|c|}
\hline Phenotype & Prdm6 $6^{f l / f l}$ Wnt1-Cre2 & Prdm6 $6^{f l /+ \text { Wnt1-Cre2 }}$ & Prdm6 $6^{f l f l}, \operatorname{Prdm} 6^{f l /}$ \\
\hline & & Prdm6 ${ }^{+/+}$Wnt1-Cre2 & Prdm6 $6^{+/+}$ \\
\hline Closed DA & $0 / 5(0 \%)$ & $8 / 8(100 \%)$ & 13/13(100\%) \\
\hline Patent DA & $5 / 5(100 \%)$ & $0 / 8(0 \%)$ & $0 / 13(0 \%)$ \\
\hline
\end{tabular}

differentiation and diminished their subsequent contribution to the DA and myocardium, prompting investigations into molecular mechanisms of the disease.

Loss of Prdm6 impairs proper migration and differentiation of CNCCS. The reduced number of ZsGreen1-positive cells in the DA of Prdm6 ${ }^{\text {flfl }}$ Wnt1-Cre2 ZsGreen1 mice at E17.5 and P0.5 (Figure 3A and Supplemental Figure 2, A and C) suggested impaired migration and/or differentiation of CNCCs. Considering the low levels of Prdm6 transcripts, we embarked on using scRNA-Seq analysis of CNCCs at E9.5 (21). Cardiac NCCs were extracted by dissecting out Prdm6 $6^{\text {l/fl}}$ Wnt1-Cre2 ZsGreen1 embryos and their WT littermates (Prdm6 ${ }^{+/+}$ Wnt1-Cre2 ZsGreen1) from mid otic placode to the posterior edge of the fourth somite (Figure 4A). ScRNASeq was carried out using the 10x Genomics Chromium system. The quality control (QC) threshold was made using a modified Seurat pipeline based on 2 QC covariates, count depth and the number of genes per barcode (see Methods). There were an average of 2742 and 3102 counts per cell or an average of 1294 and 1432 unique genes detected in WT and KO conditions, respectively. Only samples with RNA integrity number (RIN) greater than 6.5 were used (see Methods for more details). Graph-based clustering was performed using Seurat, and the PHATE (Potential of Heat-diffusion for Affinity-based Trajectory Embedding) technique developed by Moon and van Dijk et al. (22) was used to visualize trajectory structures. Cluster annotation was made manually based on specific cell markers (Supplemental Table 1).

PHATE-based analyses revealed 31 distinct clusters (Figure 4B and Supplemental Figure 3). There was no obvious difference in the evolution of these clusters from pre-epithelial mesenchymal transition (preEMT)/EMT transition to migrating CNCCs and their terminal differentiation into cardiac and SMC lineages between WT and KO mice clusters. Although the total number of cells was similar between $P r d m 6^{\text {f/fl }}$ Wnt1-Cre2 ZsGreen1 mice and their WT littermates, the number of cells in each cluster varied between the 2 genotypes. Specifically, there were more cells in the neural tube and fewer cells in the EMT and migrating NCC clusters of Prdm6 $6^{A / f l}$ Wnt1-Cre2 ZsGreen1 mice compared with their WT littermates (Prdm6 $6^{+/+}$Wnt1Cre2 ZsGreen1) (Figure 4C), consistent with our earlier findings from imaging (Figure 2, B and C). The single-cell transcript analysis showed that Prdm6 transcript levels were reduced in 2 clusters of the Prdm6 ${ }^{\text {flfl }}$ Wnt1-Cre2 ZsGreen1 mice compared with WT mice: 1 of 2 cardiac muscle lineages (cluster 8) and pre-EMT (cluster 10) (Figure 4D). Strikingly, in cluster 8, there was reduced expression of SMC markers Tagln and Actg2 and increased expression of endothelial genes and axonal guidance, such as Sema3c, Sema6d, Chd7, and CD34 (http://betsholtzlab.org/VascularSingleCells/database.html), in Prdm6 ${ }^{\text {Alfl }}$ Wnt1-Cre2 ZsGreen1 versus WT littermates (Supplemental Figure 4), suggesting impaired differentiation of CNCCs. This was consistent with our earlier observation of ZsGreen1 and VE-cadherin coexpression in the DA of Prdm6 $6^{A / f l}$ Wnt1-Cre2 ZsGreen1 mice at P0.5 (Supplemental Figure 2, A and B). Our findings collectively indicated that loss of Prdm6 in CNCCs resulted in the presence of fewer differentiated SMCs in the DA due to impaired migration and differentiation of CNCCs.

Smooth muscle-specific Prdm6-KO mice die postnatally from isolated PDA with altered differentiation of SMCs. To investigate the effect of Prdm6 disruption on vascular smooth muscle cell (VSMC) differentiation, we crossbred SM22-Cre mice with homozygote Prdm6 $6^{A / f}$ mice to generate Prdm6 ${ }^{f /+}$ SM22-Cre mice and then

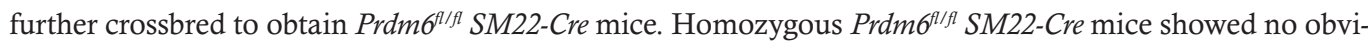
ous growth retardation or syndromic features (Supplemental Figure 5), but all died at P1.5, slightly later compared with Prdm6 $6^{\text {Ilfl }}$ Wnt1-Cre2 ZsGreen1 mice. Gross anatomical examination of the cardiovascular system showed a PDA, albeit with a smaller diameter compared with Prdm6 $6^{\text {flfl }}$ Wnt1-Cre2 ZsGreen1 mice (Figure 5A). The cross-section examination of the DA showed complete patency of DA (Figure 5B), a completely penetrant trait in both male and female mice (Table 2). We assessed cell proliferation in the DA at E17.5 using Ki67, when ductal cells were still viable in WT mice. Once again, we detected no significant 
A

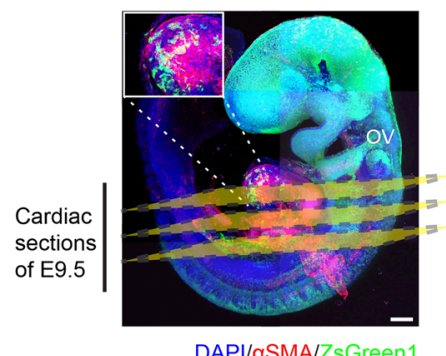

C
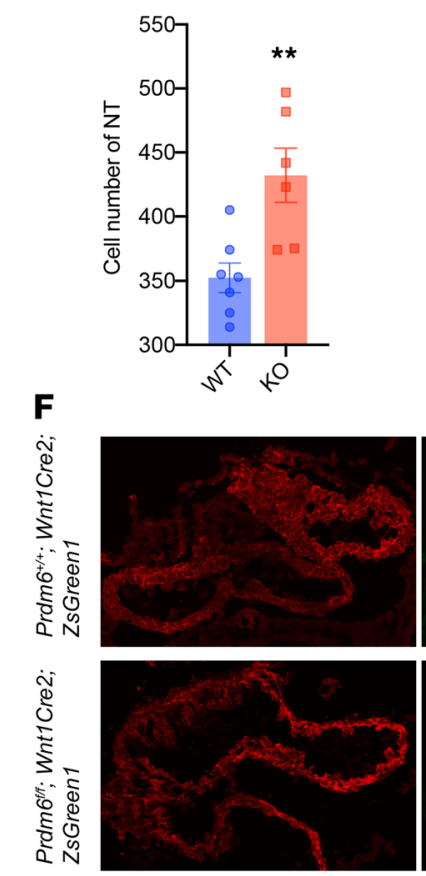

B

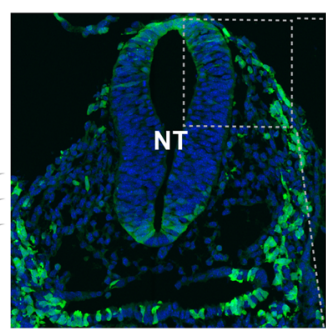

D

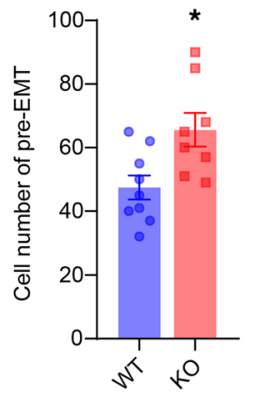

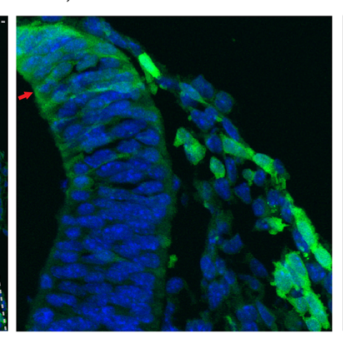

E

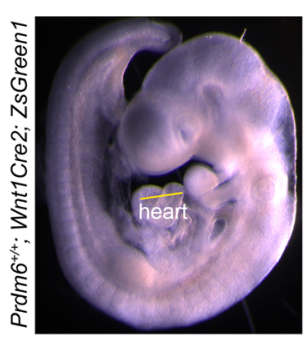

Prdm6 6ff; Wnt1Cre2; ZsGreen1
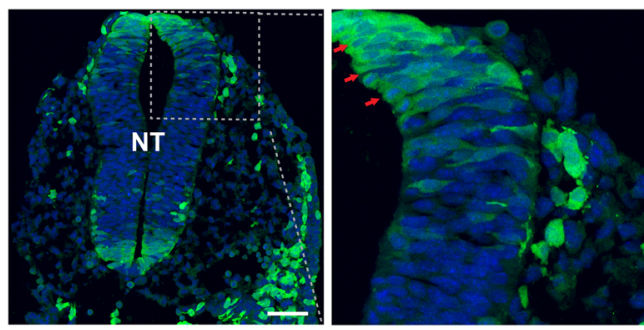

DAPI/ZsGreen
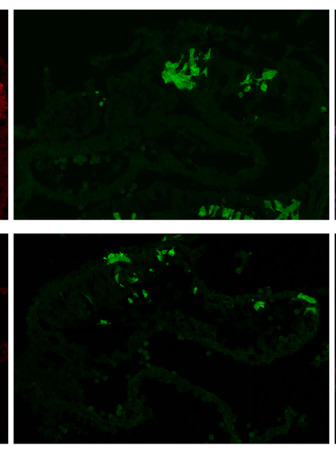
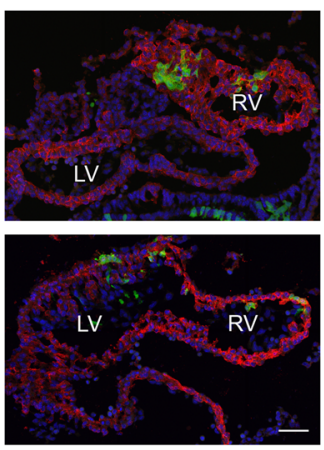

DAPI/aSMA/ZsGreen1
G
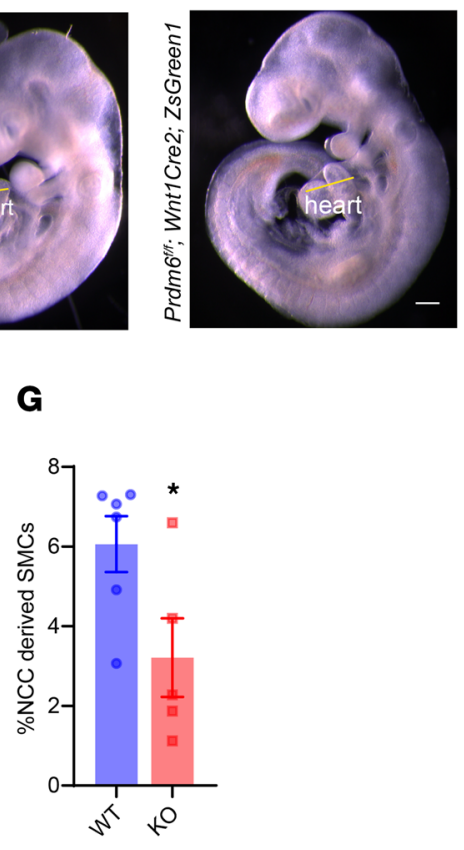

Figure 2. Fate mapping of CNCC establishes the role of Prdm6 in CNCC migration. (A) Whole-mount confocal image of Prdm6 ${ }^{+/+}$Wnt1-Cre2 ZsGreen1 E9.5 embryo. The inset shows the colocalization of $\alpha$ SMA (red) with ZsGreen1(green); nuclei are stained with DAPI (blue). Scale bar: $200 \mu \mathrm{m}$. (B) Transverse sections of cardiac neural crest at E9.5, using confocal imaging, demonstrating the retention of ZsGreen1-positive neural crest cells (green) in the neural tube (red arrow) in Prdm6 ${ }^{f / f l}$ Wnt1-Cre2 ZsGreen1 versus littermate controls (Prdm6 $6^{f+/}$ Wnt1-Cre2 ZsGreen1); nuclei are stained with DAPI (blue), scale bar: $50 \mu \mathrm{m}$. (C and D) Quantification of total neural tube and pre-EMT cells, respectively. (E and F) Cross-sections of E9.5 myocardium using confocal imaging, showing thinner myocardium and lesser CNCC contribution to myocardium in Prdm6 $6^{f / f}$ Wnt1-Cre2 ZsGreen1 (bottom) versus controls (Prdm6 $6^{f+/}$ Wnt1-Cre2 ZsGreen1, top); $\alpha$ SMA (red), DAPI (blue), and ZsGreen1 (green). LV, left ventricle; RV, right ventricle. Scale bar: $50 \mu \mathrm{m}$. (C) Percentage of NCC-derived smooth muscle cells (SMCs) in myocardium. The image intensities were quantified by Imagej; the thresholds for positive color detection were kept constant between different images. Each dot represents a biological replicate. The comparison between different groups was done by a 2-tailed unpaired $t$ test, and data are shown as mean \pm SEM. ${ }^{*} P<0.05,{ }^{*} P<0.01$. All controls were the corresponding littermates ( $n=5-9$ per group).

difference in Ki67 staining between Prdm6 ${ }^{1 / / 7}$ SM22-Cre and WT littermates (Figure 5, C and D). This was a striking finding given the patency phenotype and suggested that the loss of Prdm6 in VSMCs impairs their differentiation but not proliferation. Consistent with this, bulk RNA-Seq analysis of E17.5 DA from Prdm$6^{\text {I/f }}$ SM22-Cre mice and WT littermates identified contractile proteins, such as Tagln and Myh11, as the most downregulated genes in the DA of Prdm6 ${ }^{\text {H/l }}$ SM22-Cre mice versus WT littermates (Figure 5E and Supplemental Figure 6) from 280 differentially expressed genes (Supplemental Table 2). Strikingly, the expression levels of Tfap $2 b$ and Sox9, which also function as NCC specifiers, were significantly reduced in the DA of $P r d m 6^{\text {Alf }} S M 22-C r e$ versus WT littermates (Figure 5E and Supplemental Figure 6). Real-time quantitative PCR (qRT-PCR) further confirmed the reduced expression of Prdm6 (Figure 6A) in both the DA and aorta. However, the transcript levels of contractile proteins Myh11 and Tagln were reduced in the DA and not the descending aorta of Prdm6 $6^{\sharp / f}$ SM22-Cre mice at E17.5 (Figure 6, B and C). The expression levels of Tfap2b and Sox9 were also decreased, whereas those of the endothelial marker $K d r$ (also known as Flk1, Vegfr2) 
A
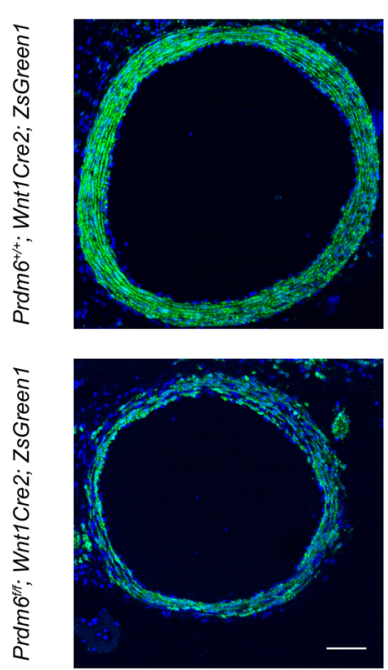

DAPI/ZsGreen

C
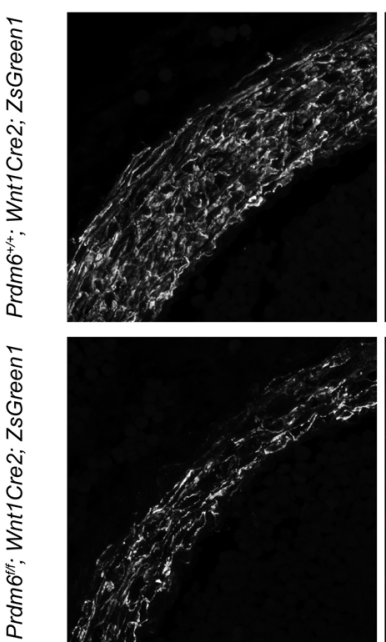

D

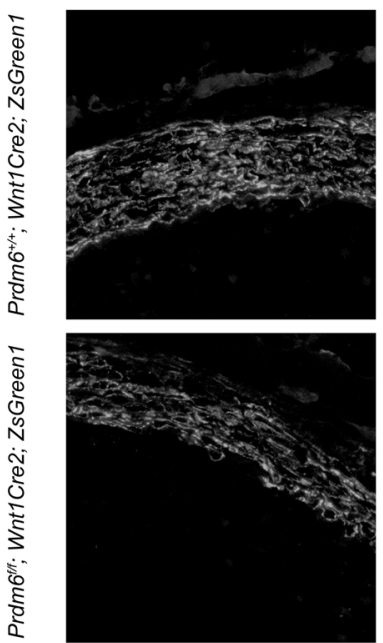

B

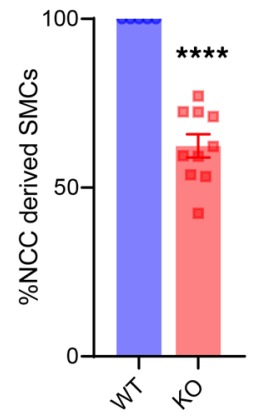

E

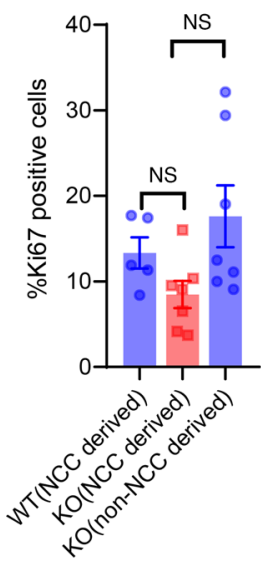

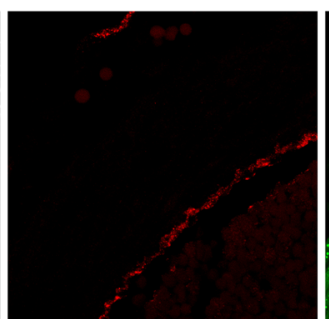
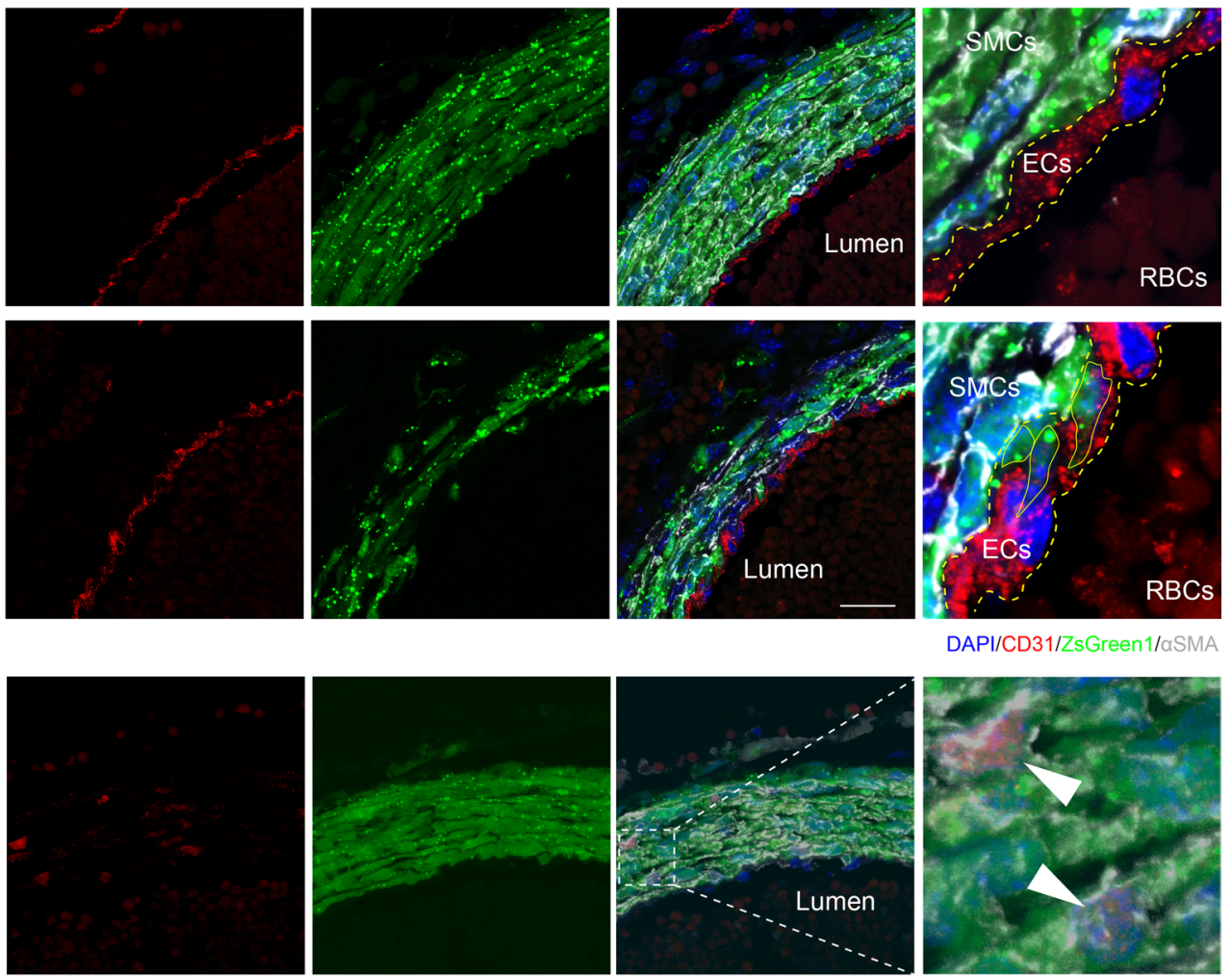

DAPI/CD31/ZsGreen1/aSMA
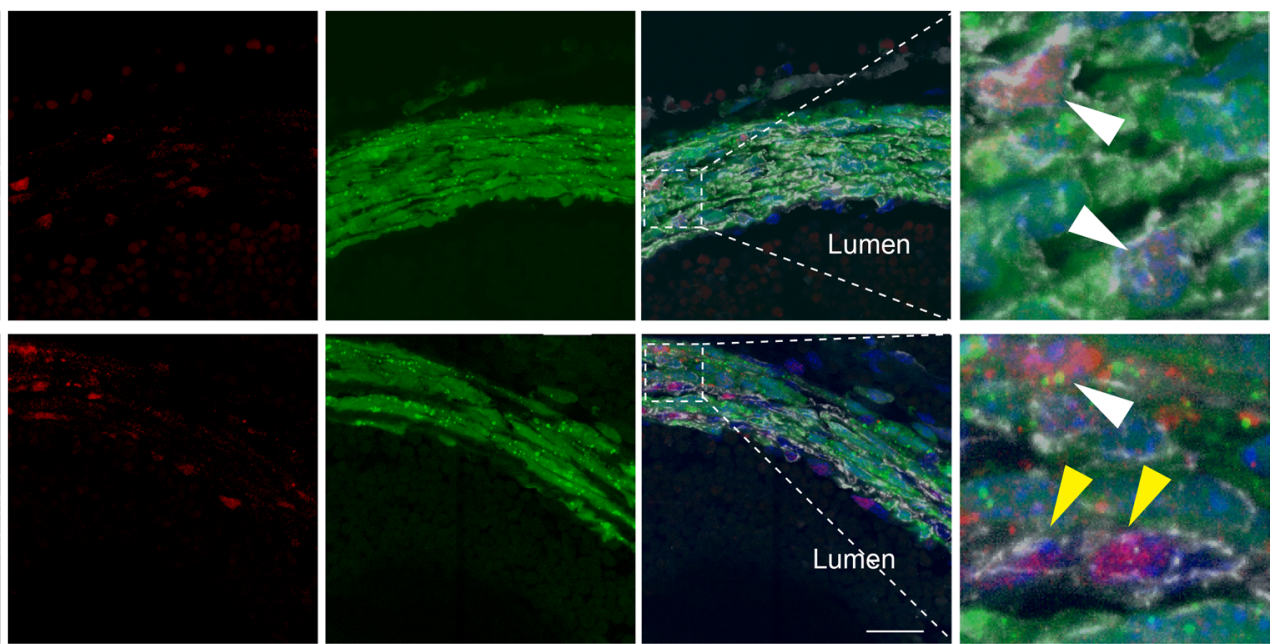

DAPI/Ki67/ZsGreen1/aSMA

Figure 3. Fate mapping of CNCCs in neural crest-specific Prdm6-deficient mice shows their reduced contribution to ductus arteriosus. (A) Representative cross-sections of DA of WT (top) and Prdm6 fl/fl Wnt1-Cre2 ZsGreen1 (bottom) DA at E17.5 by confocal imaging; scale bar: $50 \mu \mathrm{m}$. (B) Percentage of neural crest-derived SMCs. (C) Magnified image demonstrating the colocalization of $\alpha$ SMA (gray) and ZsGreen1 (green). In contrast to controls, the DA wall was thinner and only a fraction of SMCs of Prdm6 f//fl Wnt1-Cre2 ZsGreen1 were NCC derived. In addition, the NCC-derived SMCs of Prdm6 $6^{f / f l}$ Wnt1-Cre2 ZsGreen1 have an irregular shape (outlined by straight yellow lines) and have infiltrated and disrupted the endothelial layer, stained with CD31 antibody (outlined by dotted yellow lines); scale bar: $20 \mu \mathrm{m}$. (D) The confocal images of representative cross-sections of DA of control (top row) 
and Prdm6 fl/fl Wnt1-Cre2 ZsGreen1 (bottom row) mice at E17.5 stained for Ki67 (red) and $\alpha$ SMA (gray) show the localization of ZsGreen1 (green) and Ki67 (white arrowheads) and Ki67 in ZsGreen1-negative SMCs, indicated by yellow arrowheads. Scale bar: 50 mm. (E) Percentage of Ki67-positive cells in NCC-derived SMCs in WT mice and NCC-derived and non-NCC-derived SMCs in Prdm6flfl Wnt1-Cre2 ZsGreen1 (KO) DA. All SMCs in WT mice were NCC derived. There was no significant difference in the percentage of Ki67-positive NCC-derived SMCs between WT and KO mice. The image intensities were quantified by Imagej, and the thresholds for positive color detection were kept constant between different images. Each dot represents a biological replicate. The comparison between different groups was done by a 2-tailed unpaired $t$ test, and data are shown as mean \pm SEM. The comparisons between multiple groups (E) were done by 1-way ANOVA. A Mann-Whitney test was conducted for non-normally distributed data. ${ }^{* * *} P<0.0001$. All controls were the corresponding littermates ( $n=5-10$ per group).

were increased at E17.5 and P0.5 in the DA (Figure 6, D-F). These findings further support the role of Prdm6 as a regulator of SMC differentiation during cardiovascular development. Although Prdm6 ${ }^{\text {flfl }}$ SM22 Cre mice had smaller DA size and lumen compared with Prdm6 $6^{\prime / f l}$ Wnt1-Cre2 ZsGreen 1 mice, they died from PDA, signifying the importance of SMC differentiation in DA closure.

To identify the Prdm6-regulated gene network, we applied gene set enrichment analysis by using Gene Ontology (GO). Two separate analyses using genes significantly upregulated and downregulated were carried out. Vasculature development, blood vessel development, smooth muscle tissue development, collagen fibril organization, extracellular matrix assembly, and cell-matrix adhesion were the most significantly downregulated, and cell-cell adhesion and negative regulation of cell differentiation were among the most significantly upregulated pathways (Supplemental Table 3). We assessed the protein levels of selected differentially expressed genes by immunofluorescent staining of the DA of Prdm6 ${ }^{l / f l} S M 22$-Cre versus WT littermates at E17.5. We found that the SMC contractile protein Myh11 and fibronectin, a protein synthesized and secreted from SMCs, were significantly reduced in the DA of Prdm6 $6^{\text {Alfl }}$ SM22-Cre versus WT littermates (Supplemental Figure 7, A and B). Fibronectin has been shown to be synthesized to a significantly greater degree in the DA compared with the aorta at early gestation and contributes to the enhanced migration of DA SMCs (23).

Impaired CNCC migration in Prdm6 $6^{f l f l}$ mice results from deregulated Wnt1 signaling. The induction and subsequent specification of NCCs involves the combinatorial input of multiple signaling pathways. WNT and BMP signaling are key inducers of the neural crest, but their role in CNCC fate and migration has not been well studied. We found that expression levels of Wnt1 were highest in the pre-EMT cluster (cluster 10), followed by 1 of the 4 neural tube clusters (cluster 14), and dramatically reduced after NCC delamination from the neural tube (Supplemental Figure 8A). Wnt1 transcript levels were higher in Prdm6 $6^{\text {flfl }}$ Wnt1-Cre2 ZsGreen1 versus WT littermates (Wnt1-Cre2 ZsGreen1) in several clusters, including the pre-EMT cluster (Figure 7A), which suggested that Prdm6 may function to suppress Wnt1 during emergence of CNCCs. Pathway analysis of the pre-EMT cluster showed the "Development positive regulation of Wnt/ $\beta$-catenin signaling at the receptor level" as one of the most significantly upregulated pathways (Supplemental Table 4).

Bmp signaling has been implicated in maintaining NCC identity by counteracting the effect of Wnt signaling (24). Accordingly, we found reduced levels of Bmp4 in the neural tube, cardiac muscle lineages, and SMC clusters upon loss of Prdm6 compared with littermate controls (Figure 7B, black arrowheads; Supplemental Figure 8B), concomitant with increased Wnt1 levels. This indicated that Prdm6 controlled a balance between BMP/Wnt signaling, which was impaired in CNCCs of Prdm6 $6^{\sharp / f l}$ mice and impeded their delamination and migration.

To establish the role of Prdm6 in NCC migration, we cultured excised neural tubes of the E9.5 WT and Prdm6 ${ }^{\text {Alfl }}$ Wnt1-Cre2 ZsGreen1 embryos ex vivo to assess migration of CNCCs. We observed a marked reduction of NCC migration after 24 hours in Prdm6 $6^{\text {llfl }}$ Wnt1-Cre2 ZsGreen1 versus WT littermates (Figure 8, A and B). Postulating that the increased Wnt1 expression underlies the CNCC migration defect, we examined whether the treatment of WT neural tube tissue ex vivo with Wnt1 could phenocopy the Prdm $6^{f / f l}$ condition. We therefore repeated these experiments using WT neural tubes and treated them with WNT1 $(100 \mathrm{ng} / \mathrm{mL})$ for 24 hours. In response, we observed reduced migration of the delaminated NCCs compared with the PBS control, supporting our hypothesis (Figure 8, C and D). These findings are consistent with a prior report that constitutive activation of $\beta$-catenin in the NCC-derived SMC precursor results in a reduced NCC-derived population of SMCs in the DA (20), leading to its patency. Our findings also identified excess Wnt activation as a mechanism for PDA.

Loss of Prdm6 impairs H4K20 methylation and G1-S phase progression. We next focused on the molecular mechanisms that could link loss of Prdm6 to altered gene expression. We and others had previously shown that PRDM6 regulates H4K20 methylation $(9,25)$. We compared H4K20 monomethylation of Prdm6 $6^{\text {flfl }}$ Wnt1-Cre2 ZsGreen1 mice CNCCs at E9.5 with those of the WT littermates ex vivo, and consistent with earlier findings, we observed reduced H4K20 monomethylation in the solitary leading edge of Prdm6 $6^{t / f}$ 
A
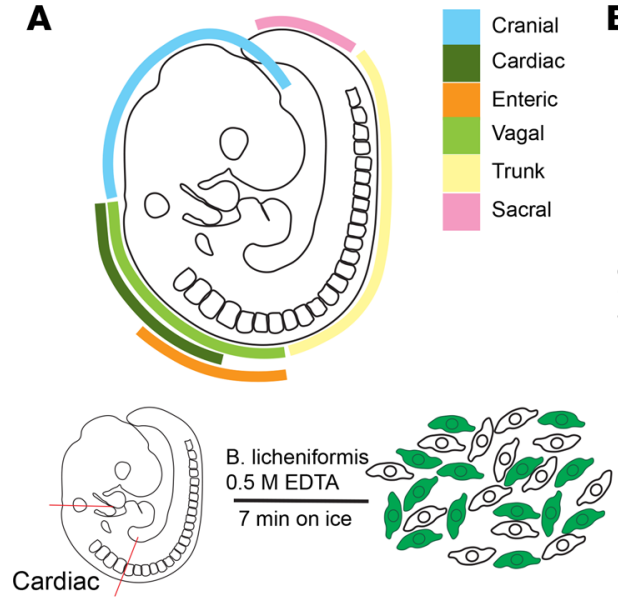

C

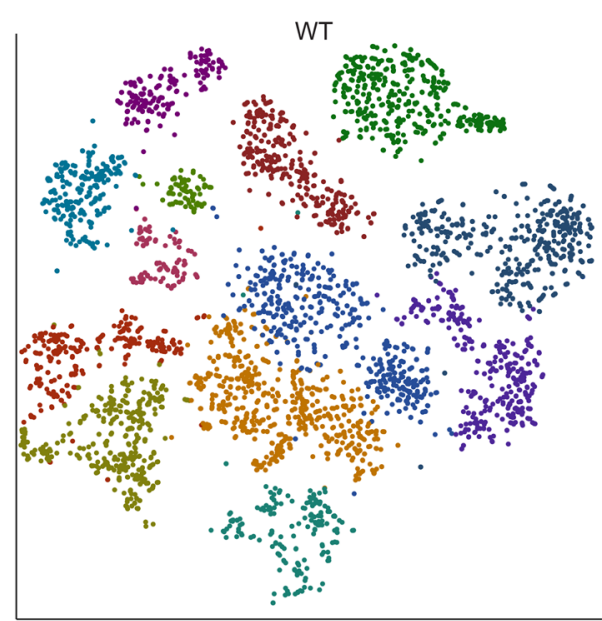

B

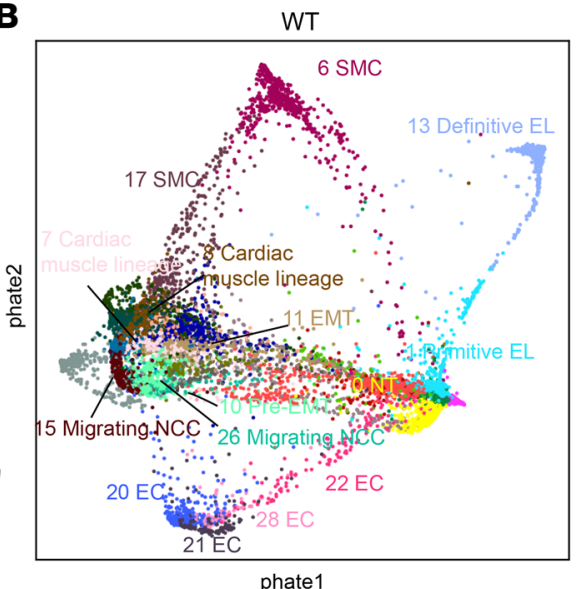

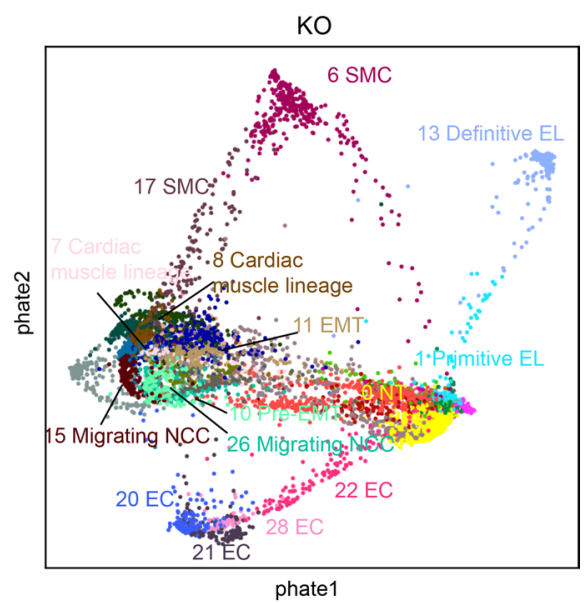

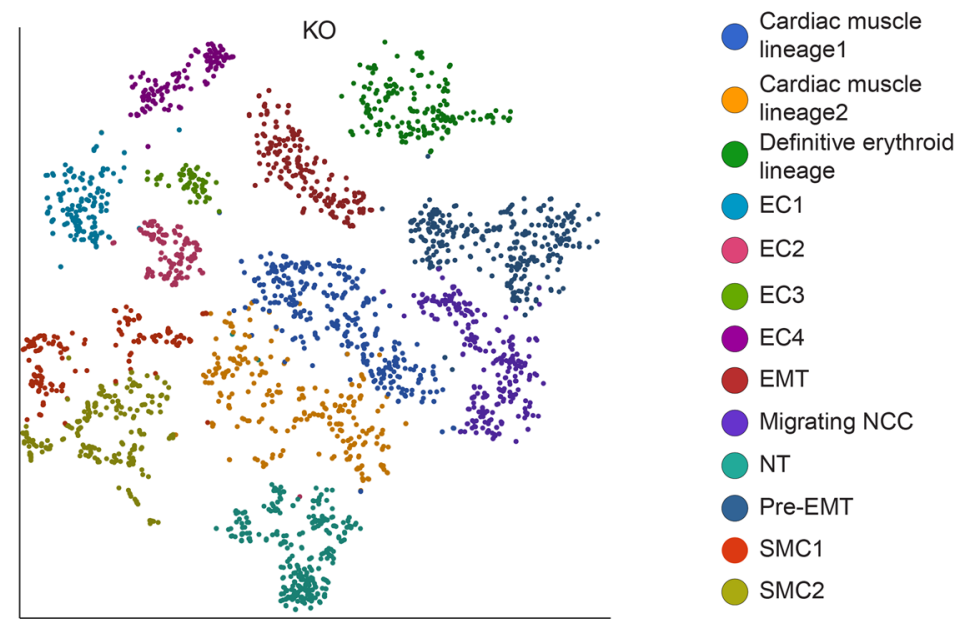

D
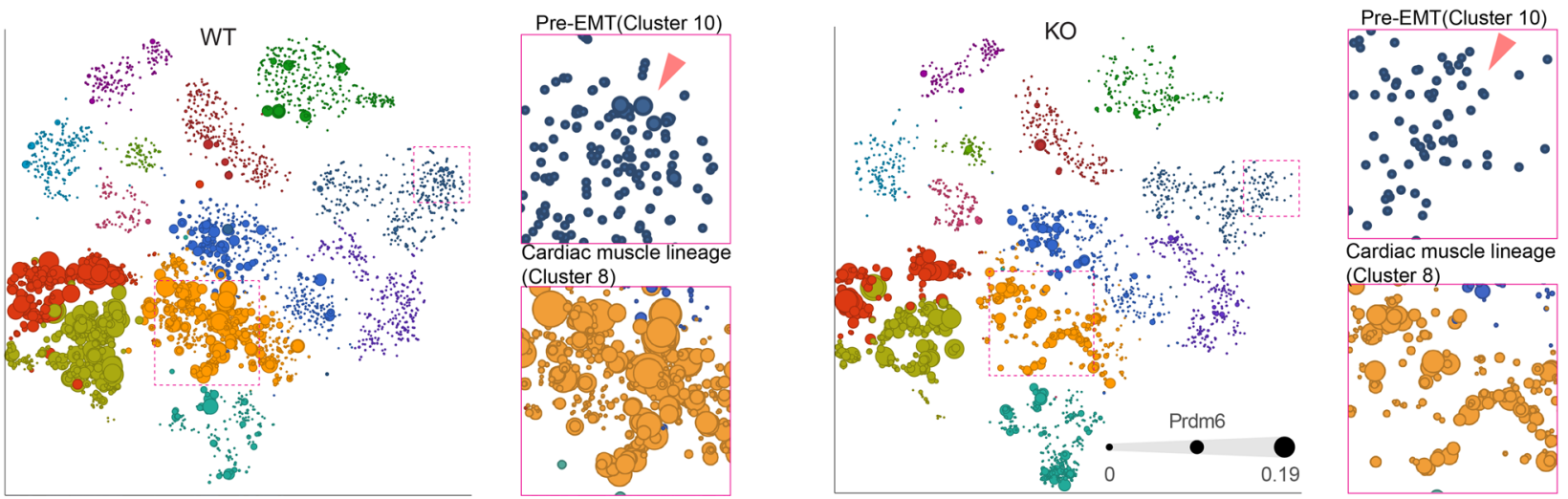

Figure 4. Single-cell RNA-Seq of the cardiac neural crest region. (A) Schematic of neural crest segments shown in different colors (top). The segment excised for single-cell RNA-Seq of CNCCs of E9.5 embryo (bottom). (B) PHATE embeddings of Wnt1-specific KO Prdm6 mice and the corresponding littermates. (C) The t-SNE plots of selected clusters of Wnt1-specific KO Prdm6 mice and the corresponding littermates generated by Partek flow. Each dot represents a cell (https://www.partek.com/partek-flow/). (D) The t-SNE plots displaying the expression levels of Prdm6 in each cluster represented by dot sizes. The insets represent the magnification of pre-EMT (blue); cardiac muscle lineage (yellow) clusters marked by lines ( $n=3$ per group).

Wnt1-Cre2 ZsGreen1 CNCCs (Figure 8, E-G). There was no significant difference of H4K20 monomethylation in early migrating NCCs (Supplemental Figure 9, A and B). Together, these results suggest that loss of Prdm6 reduced H4K20 methylation in CNCCs.

Interestingly, H4K20 monomethylation (H4K20me1) has been shown to promote $\mathrm{G} 1$ to $\mathrm{S}$ cell cycle progression (26), which is an essential step for the delamination of premigratory NCCs (27). H4K20me1 
A

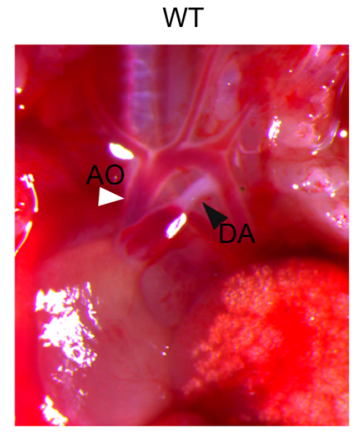

C
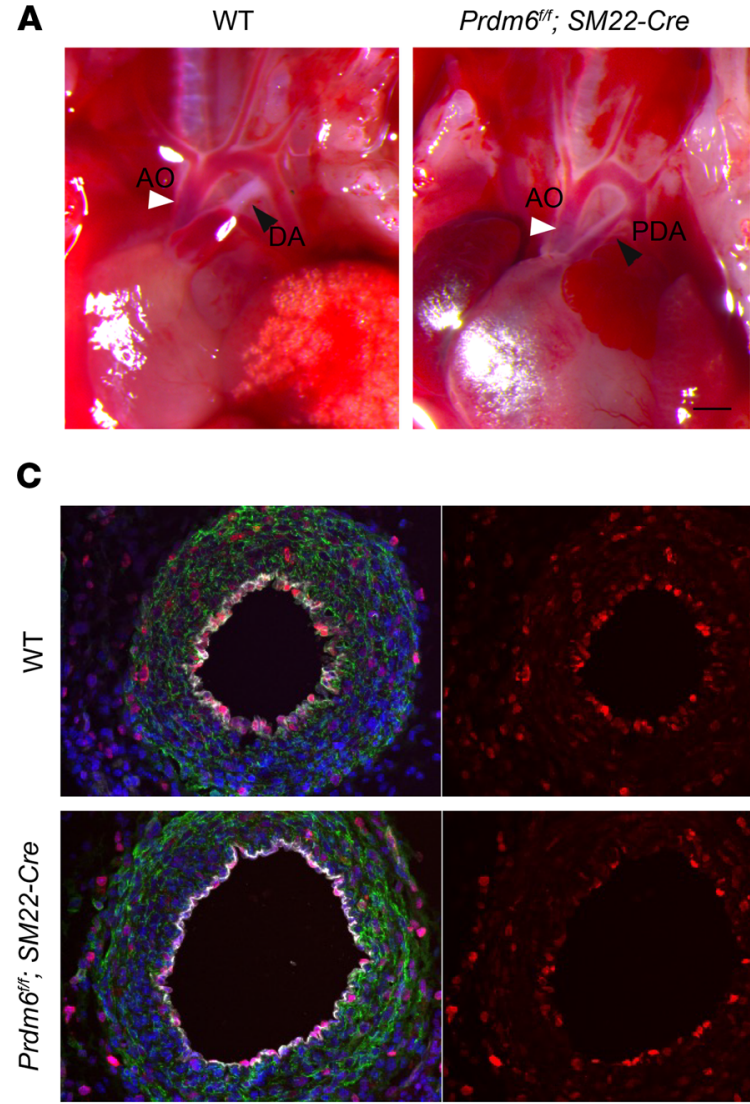

D

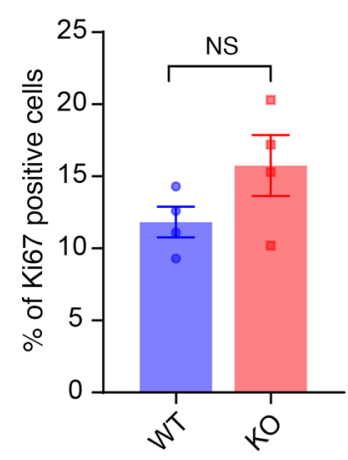

E genes: KO/WT

B
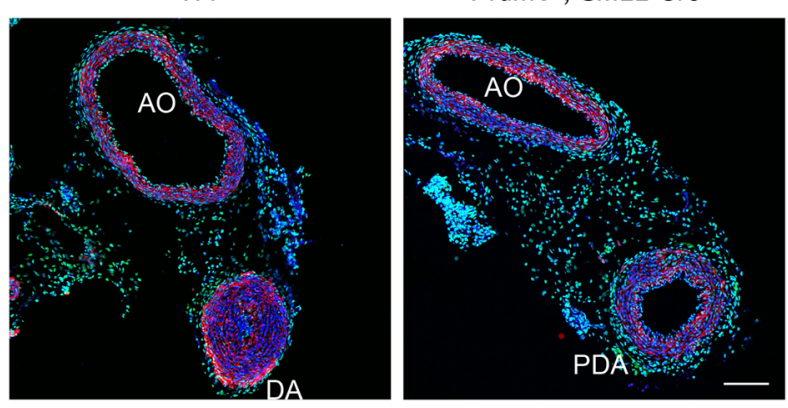

DAPI/aSMA/H3
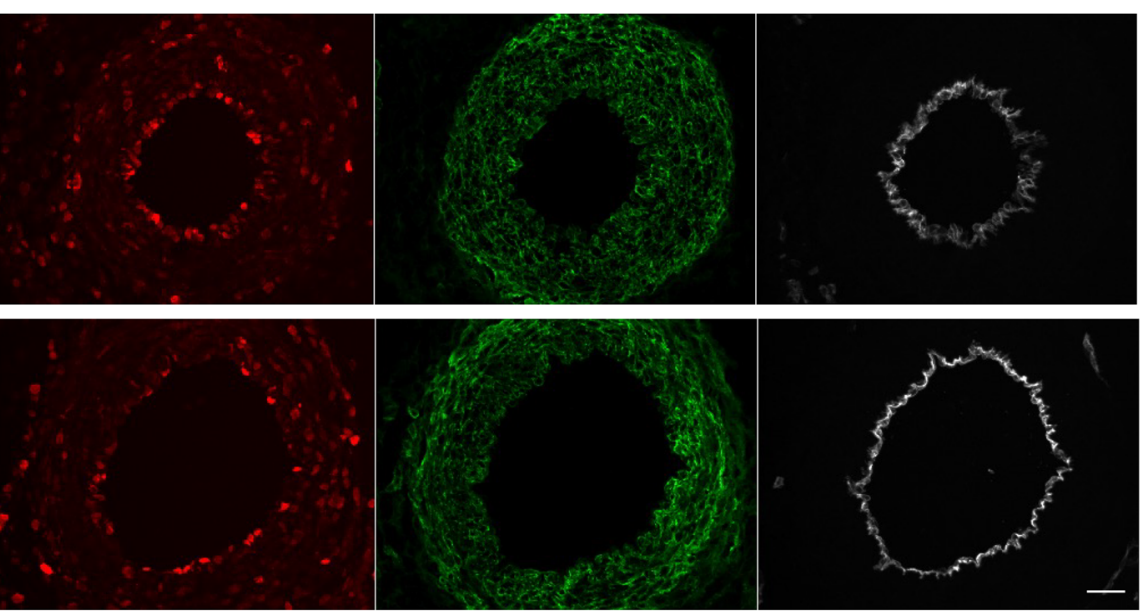

DAPI/Ki67/aSMA/CD31

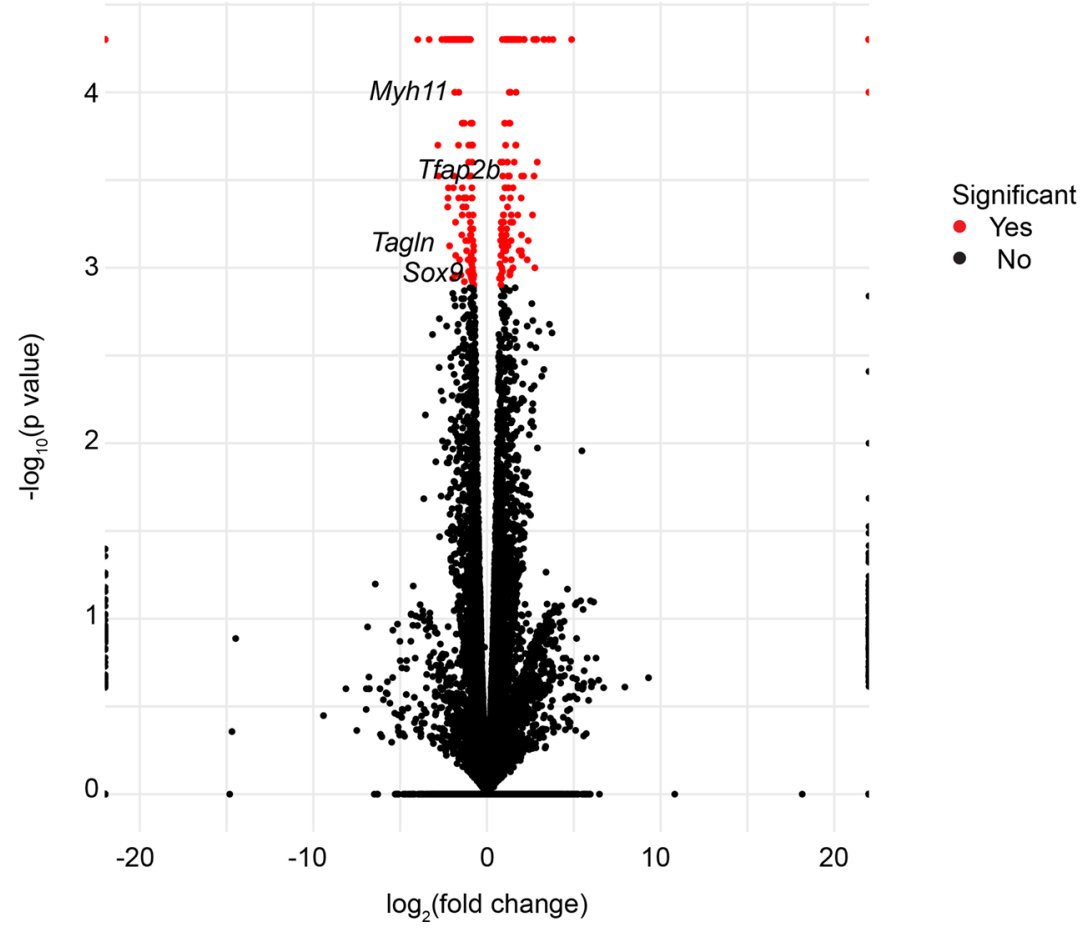

Figure 5. Smooth muscle-specific disruption of Prdm6 in mice alters transcript levels of contractile proteins and neural crest specifiers and causes patent ductus arteriosus. (A) The heart and great cardiac vessels in control (left) and Prdm6 $6^{f / f}$ SM22-Cre pups at P0.5 (right); aorta is shown by white arrowheads and the closed DA or patent DA by black arrowheads. Scale bar: $200 \mu \mathrm{m}$. (B) The confocal images of representative cross-sections of the aorta and DA of control (left) and Prdm6 6//f SM22-Cre (right) P0.5 pups, immunostained for $\alpha$ SMA (red), histone3 (green), and DAPI (blue). Scale bar: $100 \mu$ m. (C) The confocal images of representative cross-sections of DA of control (top) and Prdm6 ${ }^{f / f l}$ SM22-Cre embryos (bottom) at E17.5 immunostained for Ki67 
(red) and costained with antibodies against $\alpha$ SMA (green), CD31 (gray), and DAPI (blue). Scale bar: $50 \mu \mathrm{m}$. (D) Quantification of Ki67-positive cells in the smooth muscle cell layer. (E) Volcano plot of bulk RNA-Seq data of Prdm6 fl/fl SM22-Cre mice DA compared with control mice at E17.5. The log fold change and the $\log P$ values are shown on $x$ and $y$ axes, respectively. The comparison between different groups was done by a 2-tailed unpaired $t$ test, and data are shown as mean \pm SEM. The significantly changed transcripts in Prdm6 fl/fl SM22-Cre versus control mice are shown in red and the locations of specific genes of interest are shown. The significantly altered transcripts were determined using the following thresholds: adjusted $P<0.05$, unpaired $t$ test, FDR $<0.05$, Benjamini-Hochberg corrected. All controls were the corresponding littermates ( $n=4$ per group).

at E- and N-cadherin promoters has also been shown to regulate their transcription and mediate EMT (28). We therefore hypothesized that H4K20me1 by Prdm6 promotes cell cycle progression and alters the expression of a gene network that is necessary for delamination and migration of the CNCCs. To test this, we used CRISPR/Cas9-based gene editing to inactivate Prdm6 in exon2 of O9 mouse NCCs in vitro. We first differentiated these cells into SMCs (Figure 9A) and verified their competency in expressing Prdm6, contractile proteins Myh11 and Tagln, and NCC specifiers Sox9 and Tfap $2 b$ at days 0, 1, 3, and 6 (Figure 9B). Subsequently, we infected the cells with lentivirus containing CRISPR/Cas9 and transfected them with either control vector or 3 different sgRNAs targeting Prdm6 exon2 (Figure 9C) and generated 3 clonal cell lines with 8, 55, and 56 bp deletions (Figure 9D). qRT-PCR expression analysis confirmed the loss of Prdm6 expression and subsequent downregulation of contractile proteins Myh11 and Tagln and NCC specifiers Tfap $2 b$ and Sox9, as well as upregulation of the endothelial maker $K d r$ in all Prdm6-deficient lines (Figure 9, E-J), consistent with our in vivo gene expression analyses. We then subjected the Prdm6-deficient CNCCs to FACS analysis to assess the cell cycle activity and observed a marked reduction in G1-S cell cycle progression compared with WT O9 cells (Figure 10A). Our analyses revealed a greater number of Prdm6-deficient CNCCs in the G1 phase and fewer in the S and G2/M phase compared with WT CNCCs, indicating G1-S phase arrest. This finding was associated with increased Wnt1 expression in Prdm6-KO versus WT O9 cells (Figure 10B).

To determine the direct role of $\mathrm{H} 4 \mathrm{~K} 20 \mathrm{me} 1$ in regulating CNCC cell cycle progression, we inactivated retinoblastoma-associated protein $\mathrm{Rb} 1$ in $\mathrm{O} 9$ cells using $\mathrm{Rb} 1$-specific siRNA. RB1 protein plays a critical role in recruiting H4K20 methyltransferases, such as Suv4-20h1/KMT5B and Suv4-20h12/ KMT5C29, and mediates mono-, di-, and tri-methylation of H4K20 (29). Knockdown of Rb1 in O9 cells resulted in modest but persistently reduced G1-S progression compared with control siRNA, as determined by FACS analysis (Figure 10C). There was an inverse relationship between G1-S progression and Wnt1 expression ( $\mathrm{R}=0.83$; Figure $10 \mathrm{C})$.

Our data suggested that loss of Prdm6 reduced H4K20me1 and increased Wnt 1 levels, impeding CNCC migration, but the order of the events remained unknown. We examined the effect of Wnt 1 on histone modifications in these cells and found that it increased H4K20me1 levels compared with PBS, as predicted (Figure 10, D and E). This finding suggests that reduced H4K20me1 after Prdm6 knockdown is not caused by increased Wnt1. Accordingly, treatment of Prdm6-deficient O9 cells with Wnt inhibitor (IWP-4, $5 \mu \mathrm{M}, 04-0036$ ) rescued the migration-defective phenotype (Figure 10, F and G); however, it did not change H4K20me1 (Figure 10H). In conclusion, we suggest a model whereby Prdm6H4K20me1 synchronizes the cell cycle, which is necessary for the regulation of Wnt1 transcript levels and CNCC migration. In contrast, the loss of Prdm6 results in reduced H4K20 monomethylation and G1-S cell cycle progression, resulting in increased Wnt1 expression and impaired CNCC migration.

\section{Discussion}

NCCs give rise to a diverse cell lineage and therefore are increasingly gaining attention for their potential role in tissue regeneration. The mouse phenotyping and fate mapping in our study showed that Prdm6 regulates differentiation and migration of CNCCs, which are the precursor of SMCs in the tunica media of the DA and pharyngeal arches (16). Consequently, loss of Prdm6 results in early postnatal death from PDA (30-32). The importance of CNCCs in DA closure has been previously shown by the ablation of myocardia in early postmigratory NCC derivatives, albeit the exact molecular mechanisms remain to be elaborated (32). Mutations in histone modifiers KMT2D (histone-lysine $N$-methyltransferase 2D) and KDM6A (lysine-specific demethylase 6A) (33) and EHMT1 (euchromatic histone-lysine N-methyltransferase 1) (34) have also been associated with syndromic congenital heart disease due to defects in NCC development (35). However, in contrast to PRDM6 mutations, defects in these genes appear to affect both cardiac and cranial NCCs. 
Table 2. Genotype and phenotype of P1 pups

\begin{tabular}{lccc}
\hline Phenotype & Prdm6 $^{f l / f l}$ SM22-Cre & Prdm6 $^{f l /+}$ SM22-Cre & Prdm6 \\
Closed DA & $0 / 12(0 \%)$ & $10 / 10(100 \%)$ & $33 / 33(100 \%)$ \\
Patent DA & $12 / 12(100 \%)$ & $0 / 10(0 \%)$ & $0 / 33(0 \%)$ \\
7 litters were counted & & & \\
\hline
\end{tabular}

Our investigations ex vivo demonstrated that increased $W n t 1$ underlies impaired CNCC migration in Prdm6-deficient mice, which was rescued by Wnt inhibition. These findings are consistent with a prior report that constitutive activation of $\beta$-catenin (ctnnb1 $\Delta$ ex3) results in a reduced NCC-derived population of SMCs in the DA and early postnatal death (20). Although NCC migration in ctnnb1 $\Delta$ ex3 was not explored, our fate mapping and functional studies suggest that excessive Wnt activation can result in impaired CNCC migration.

Our comprehensive genomic and epigenomic approach identified a Prdm6-dependent gene regulatory network that underlies the regulation of a subset of CNCCs during development. The neural crest gene regulatory networks consist of multiple complex subnetworks that are sequentially activated to control the key steps of neural crest formation, delamination, migration, and differentiation. Of particular importance is the mutually inhibitory crosstalk between Wnt and Bmp signaling in that Bmp counteracts proliferation promoted by Wnt while Wnt antagonizes BMP-dependent neuronal differentiation (36). The coordinated actions of these 2 pathways result in activation of transcriptional cascades that drive EMT and migration and cooperate with signaling systems that induce cell differentiation (37). In our models, we showed that the absence of Prdm6 tips the balance in favor of Wnt1 by reducing Bmp4 and increasing Wnt 1 transcripts in several clusters, leading to impaired CNCC migration.

PRDM6 has been previously shown by our group and others to mediate H4K20 methylation in vitro $(9,25)$. H4K20 monomethylation has been shown to be critical for cell cycle progression $(38,39)$.

A

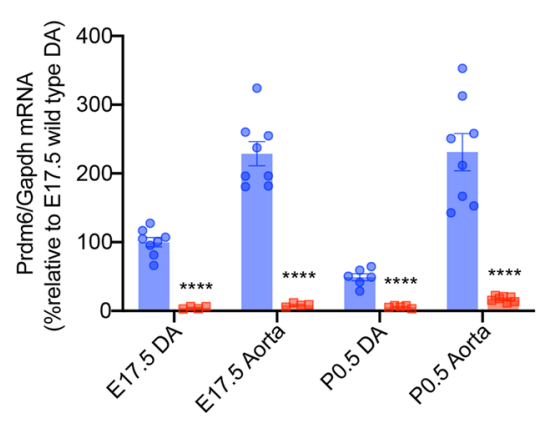

D

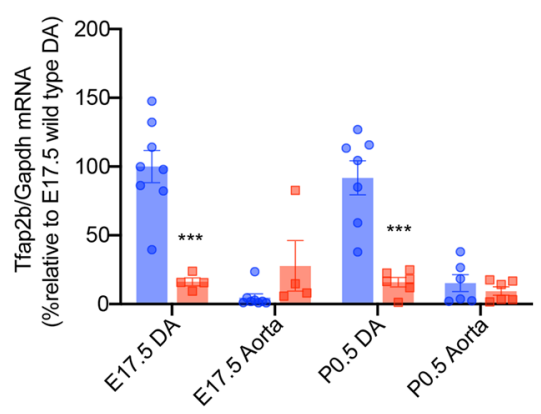

B

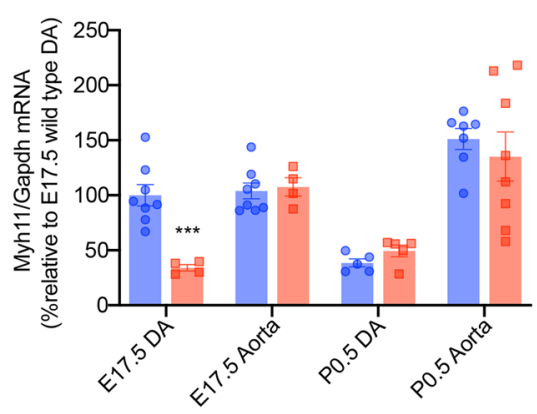

E

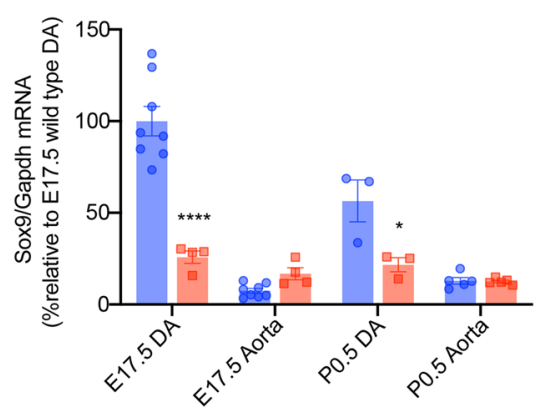

C

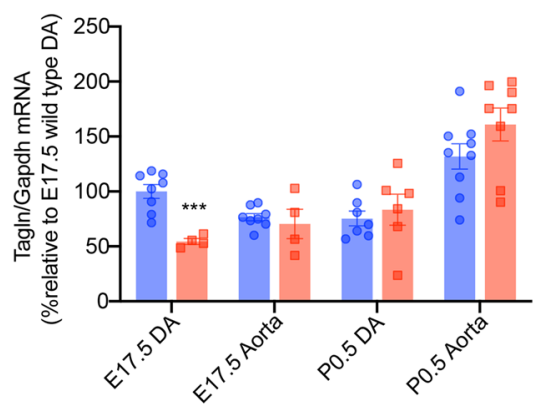

$\mathbf{F}$

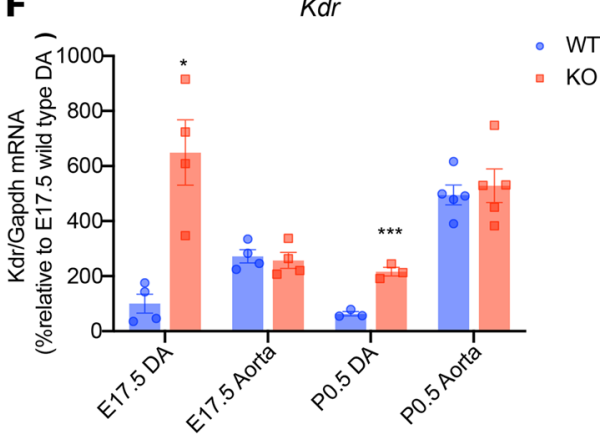

Figure 6. A representation of significantly changed transcripts in Prdm6 ${ }^{\text {flfl }}$ SM22-Cre versus control mice measured by qRT-PCR. The relative transcript levels of Prdm6 (A), Myh11 (B), TagIn (C), Tfap2b (D), Sox9 (E), and $K d r(\mathbf{F})$ in the aorta and DA of Prdm6 f/fl SM22-Cre mice versus control mice at E17.5 and $\mathrm{P0.5}$, assayed by real-time fluorescent quantitative PCR are shown. The comparison between different groups was done by a 2-tailed unpaired $t$ test, and data are shown as mean \pm SEM. Each dot represents a biological replicate. The normalcy was tested by Kolmogorov-Smirnov test. A Mann-Whitney test was conducted for non-normally distributed data. ( $n=3-9$ per group). ${ }^{*} P<0.05,{ }^{* *} P<0.001,{ }^{* * *} P<0.0001$. 
A
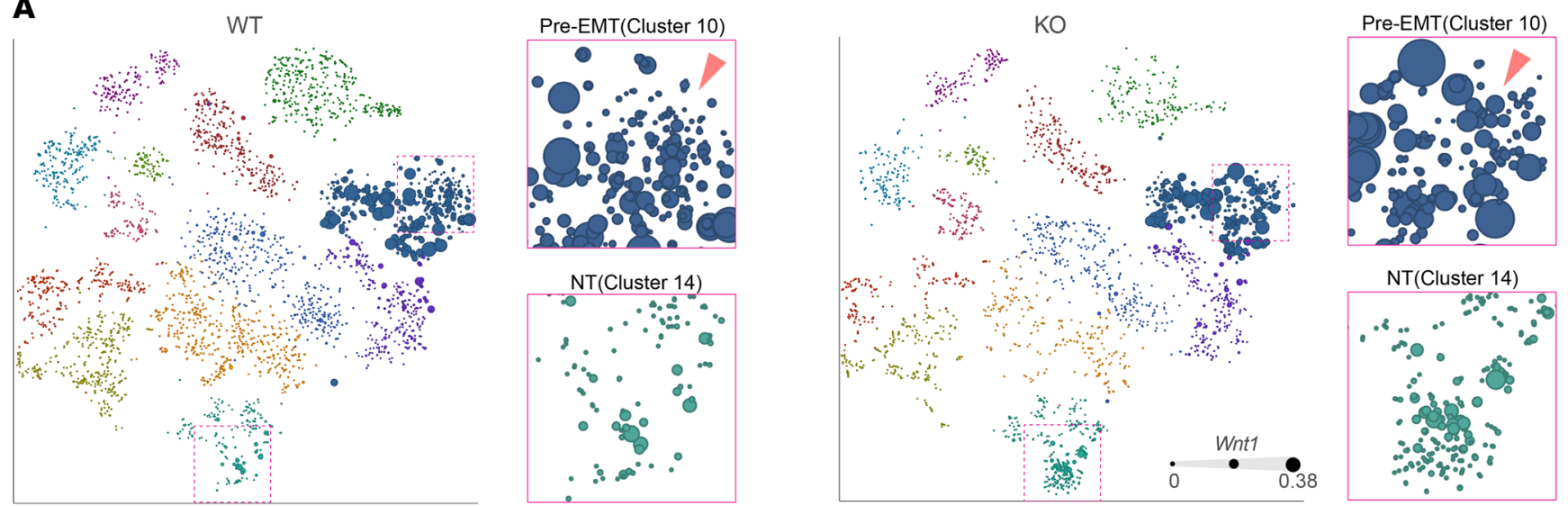

B
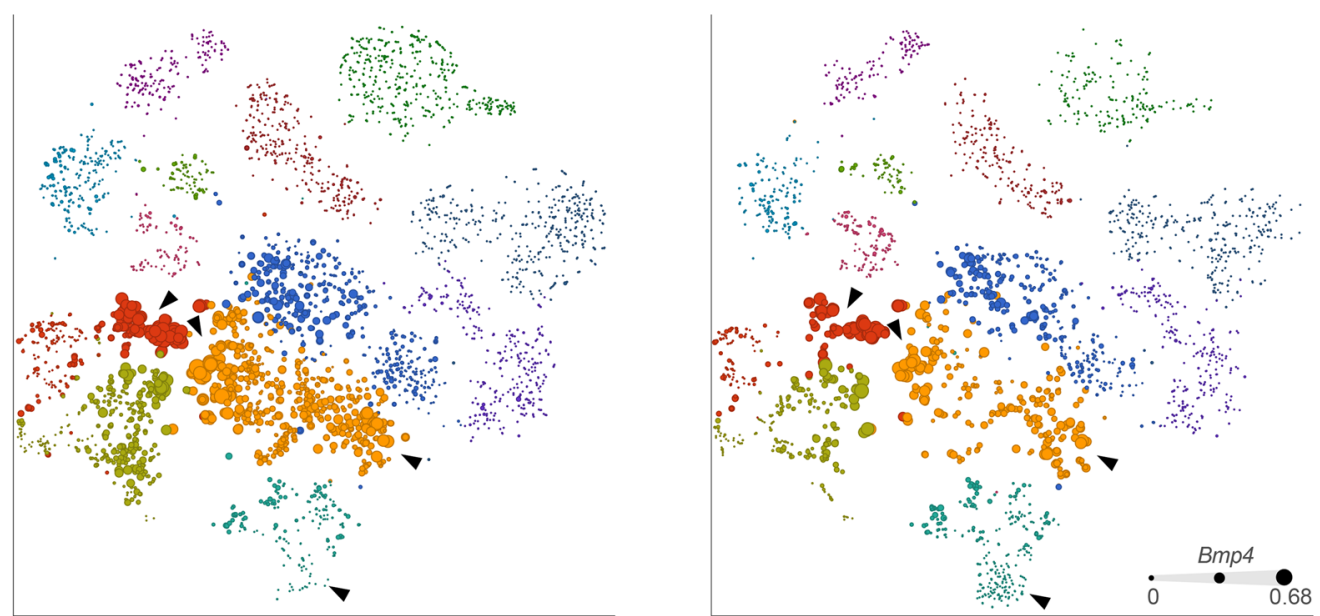

Cardiac muscle
lineage1
Cardiac muscle
lineage2
Definitive erythroid
lineage
EC1
EC2
EC3
EC4
EMT
Migrating NCC
NT
Pre-EMT
SMC1
SMC2

Figure 7. Single-cell RNA-Seq of the cardiac neural crest region of the embryo. (A) The t-SNE plots displaying the expression levels of Wnt1 in each cluster represented by dot sizes. The insets represent the magnification of pre-EMT (blue) and neural tube (green) clusters. (B) The t-SNE plots highlighting the reduced expression levels of Bmp4 in neural tube and cardiac muscle lineages of Wnt1-specific KO Prdm6 compared with the corresponding littermates. Expression levels of Bmp4 represented by dot sizes.

In the current study, we showed that Prdm6 regulates H4K20 methylation in migrating NCCs and that this event is critical for cell cycle progression and subsequent induction of EMT and migration of specific CNCCs. We showed that H4K20 methylation and cell cycle activation are inversely related to Wnt expression. The causal relationship between H4K20 methylation, cell cycle activation, and Wnt1 expression was supported by knocking down the retinoblastoma-associated protein $\mathrm{Rb} 1$, which has been shown to interact with H4-K20 methyltransferases Suv4-20h1/KMT5B and Suv4-20h12/ KMT5C (40) and to mediate their methylation of H4K20 (41). Accordingly, the knockdown of Rb1 led to G1-S phase arrest and was associated with higher Wnt1 expression. These findings linked reduced H4K20 methylation in Wnt1-Cre2 Prdm $6^{A / /}$ mice to increased Wnt1 expression in multiple CNCC clusters.

One of the most important implications of our study is the role of temporal and spatial epigenetic regulation in the specification of NCCs. It is likely in this context that elevated Wnt1 impairs a subset of CNCCs that contributes to the DA, whereas elevated Wnt1 is a necessary component of trunk NCCs, including their entry into the S-phase and their facilitated migration (42). Strikingly, environmental factors, such as teratogens and infectious disorders during the first trimester of pregnancy, have been associated with isolated persistent PDA and altered DNA or histone modifications (43-46). The characterization of Prdm6 function in NCCs has, therefore, a broad implication for the pathogenesis of inherited and acquired congenital heart diseases and may lead to identification of novel targets for drug development. 


\section{JClinsiGHT}

A

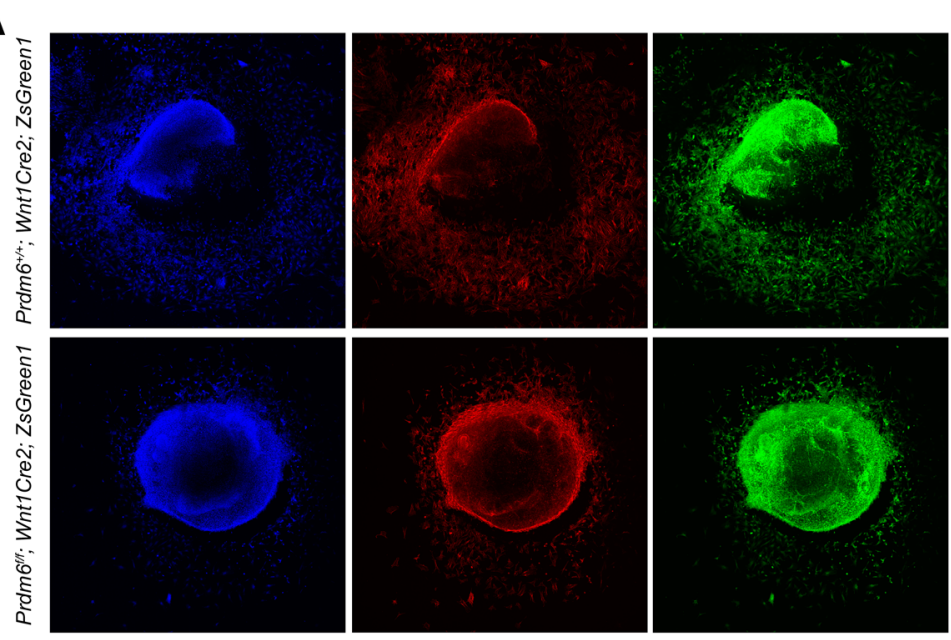

C

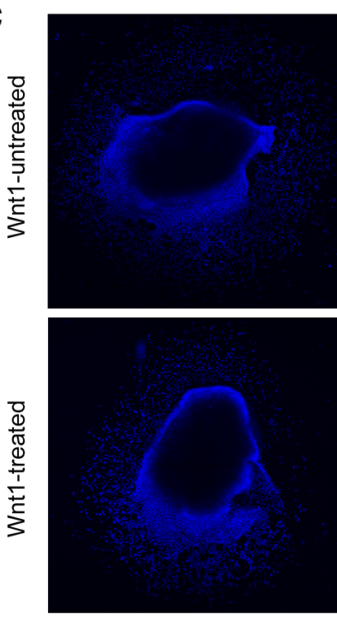

E

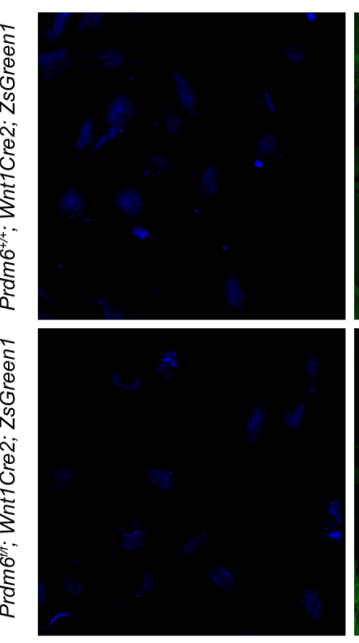

$\mathbf{F}$

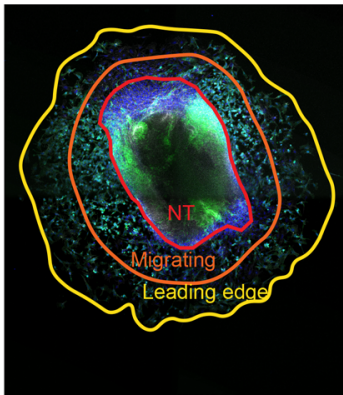

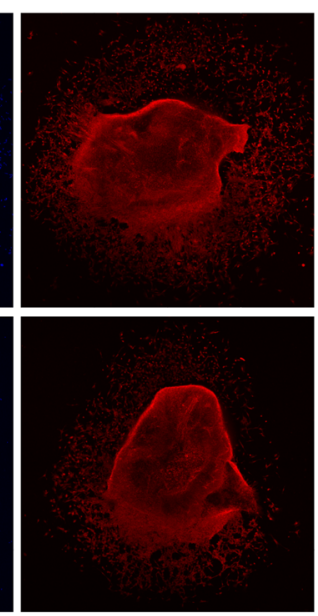
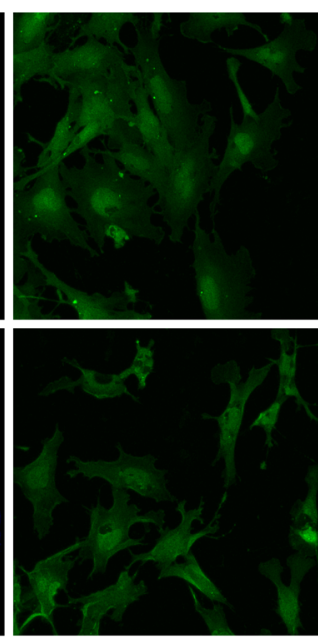

G

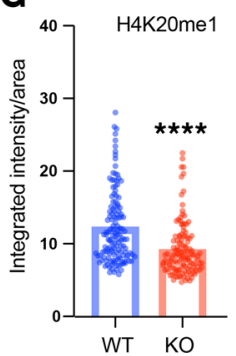

B
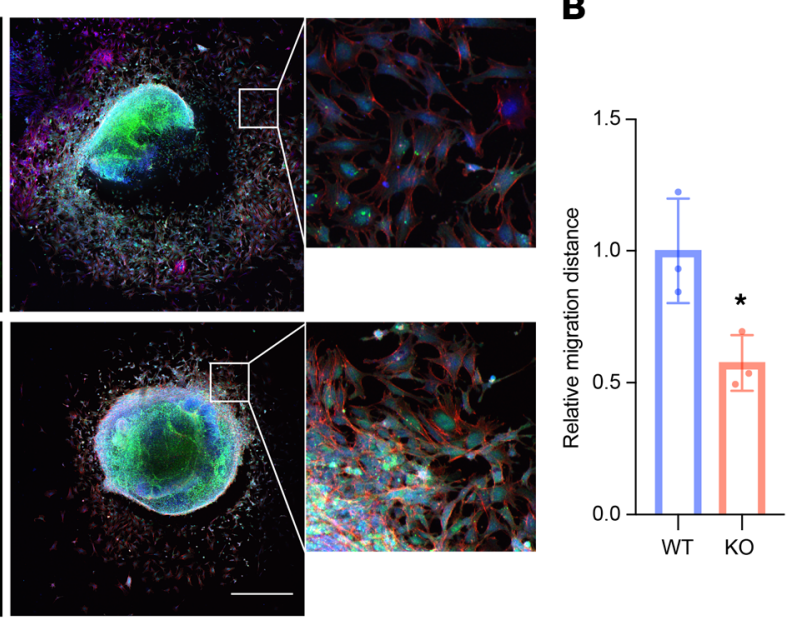

DAPI/Phalloidin/ZsGreen

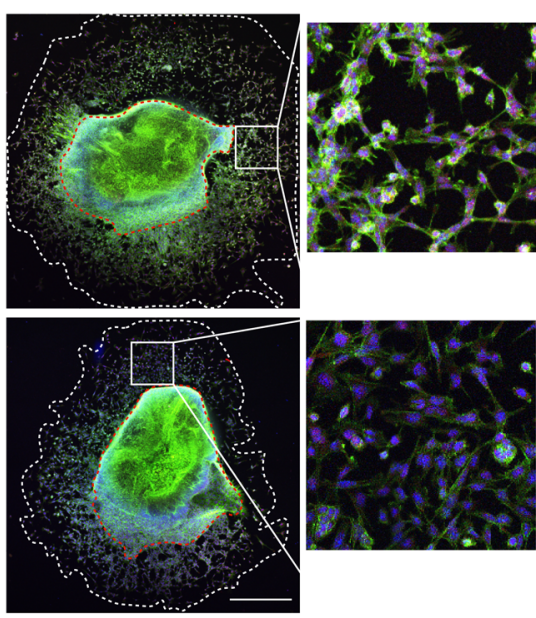

D

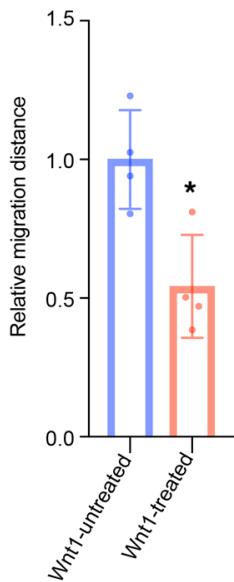

DAPI/Tfap2b/Phalloidin
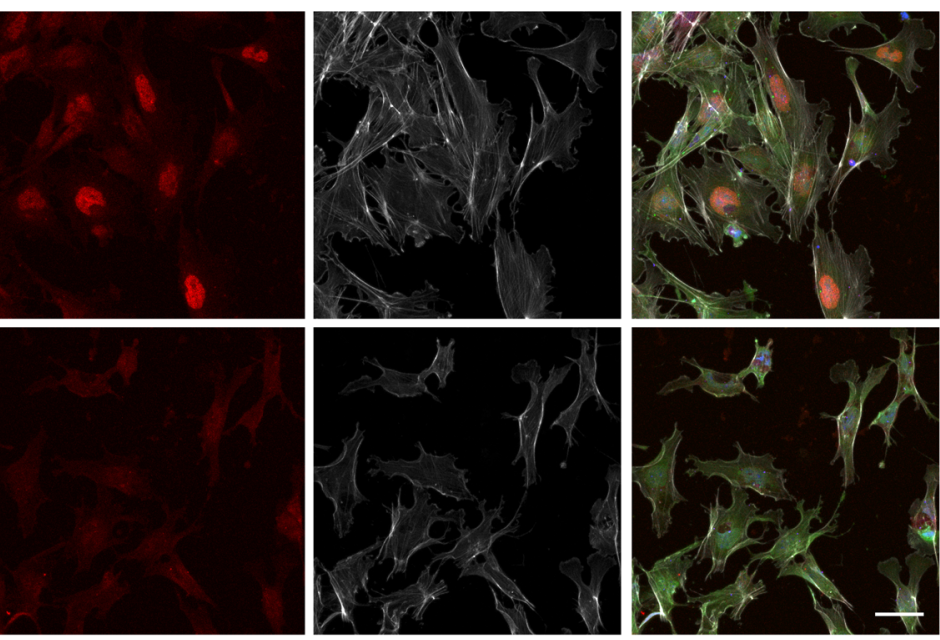

DAPI/ZsGreen1/H4K20me1/Phalloid 
Figure 8. Ex vivo examination of neural crest migration. (A) Migration of NCCs from ex vivo cultured neural tube of WT (Prdm6 $6^{+/+}$Wnt1-Cre2 ZsGreen1) and KO (Prdm6 f/fl Wnt1-Cre2 ZsGreen1) mice at E9.5. The NCCs are shown as ZsGreen1-positive (green) and the neural tube is stained with DAPI (blue) and phalloidin (red). Scale bar: $500 \mu \mathrm{m}$. (B) Quantification of migration distances; each dot represents a biological replicate. Migration is measured as distance from the periphery at the junction with 2 perpendicular radiuses. (C) Migration of NCCs from ex vivo cultured neural tube of WT mice at E9.5 treated with WNT1 for 24 hours and stained with DAPI (blue), Tfap2b antibody (red), phalloidin (green). Scale bars: $500 \mu m$. (D) Quantification data of C. Each dot represents a biological replicate. (E) H4K20 monomethylation (red) of NCCs in the leading edge of ex vivo cultured neural tube of WT (Prdm6 ${ }^{+/+}$Wnt1-Cre2

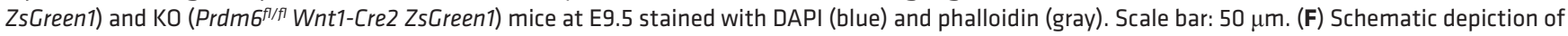
ZsGreen1-positive (green) migrating NCCs, divided into 2 populations of migrating (clustered) and leading edge (single cells). (C) Quantification of H4K20 monomethylation from $\mathbf{E}$; each dot represents a cell. The comparison between different groups was done by a 2-tailed unpaired $t$ test, and data are shown as mean \pm SEM. ${ }^{*} P<0.05,{ }^{* * *} P<0.0001$. All controls were the corresponding littermates ( $n=3$ per group).

\section{Methods}

Generation of SM22-Cre KO mice. The day of plug detection was considered as E0.5. Prdm6-flox mice were generated in-house and were intercrossed to generate homozygous Prdm6-flox mice (Prdm6 $\left.6^{A / f}\right)$. Prdm6 ${ }^{A / f l}$ mice were viable, fertile, and indistinguishable from control littermates. Prdm6 $6^{\text {Ilfl }}$ mice were intercrossed with SM22-Cre transgenic mice that expressed high levels of Cre-recombinase in SMCs (Supplemental Figure 10).

Neural crest-restricted inactivation of the Prdm6 gene. To determine the function of Prdm6 in cardiac neural crest-derived cells, homozygous $P r d m 6^{f / f l}$ mice were crossed with transgenic mice expressing Cre-recombinase under the transcriptional control of the neural crest-restricted Wnt1 promoter. Prdm6 ${ }^{t /+}$ Wnt1-Cre2 offspring were then interbred with $P r d m 6^{A /+}$ mice to generate Prdm $6^{f / f l}$ Wnt1-Cre2 mutants and control littermates Prdm6 $6^{+/+}$Wnt1-Cre2 (Supplemental Figure 11).

For lineage tracing, $P r d m 6^{f / f l}$ mice were crossed with ZsGreen1 to generate Prdm6 $\sigma^{f /+} Z s G r e e n 1$ mice and then interbred with ZsGreen1 to generate Prdm6 ${ }^{A /+} Z s$ Green1/ZsGreen1 mice. To knock out Prdm6 in Wnt1-positive NCCs, the Prdm6 $6^{A /+}$ ZsGreen1/ZsGreen1 mice were intercrossed with Prdm6 ${ }^{1 /+}$ Wnt1-Cre2 mice (Supplemental Figure 12).

Wnt1-Cre2 mice were purchased from The Jackson Laboratory, stock number 022501 (full strain name B6.Cg-E2f1Tg(Wnt1-cre)2Sor/J). ZsGreen1 mice were received from Daniel Greif (Yale University) and are also available from The Jackson Laboratory (Rosa-CAG-LSL-ZsGreen1-WPRE, Jax 007906). All mice were fed ad libitum and housed at constant ambient temperature in a 12-hour light/12-hour dark cycle. Both male and female mice were studied. All studies in animals were conducted in accordance with the NIH Guide for the Care and Use of Laboratory Animals (National Academies Press, 2011).

Mouse embryo and tissue preparation and IHC. Mouse embryos and tissues were collected and rinsed in ice-cold Dulbecco's PBS and fixed by incubation for 15-20 minutes in PBS containing 4\% PFA (Santa Cruz, sc-281692) at $4^{\circ} \mathrm{C}$. They were rinsed twice in ice-cold PBS and incubated for 24 hours in $30 \%$ sucrose $/ \mathrm{PBS}$ at $4^{\circ} \mathrm{C}$, followed by Tissue-Tek OCT compound (catalog 4583) in plastic molds and allowed to freeze on dry-ice and stored at $-80^{\circ} \mathrm{C}$. The tissue submerged in OCT compound was sectioned onto $5 \mu \mathrm{m}-10 \mu \mathrm{m}$ slices in a cryostat and dried on Fisherbrand Superfrost Plus slides (Fisher Scientific, catalog 22-037-246) 12 to 16 hours at room temperature. P0.5 mice tissue were collected after anesthesia. Hearts were then removed and treated as described above.

Immunofluorescence. All immunofluorescence staining was performed on $5 \mu \mathrm{m}-10 \mu \mathrm{m}$ frozen sections. For immunostaining, the slides were washed 3 times in $1 \times$ PBS, followed by 3 washes in PBS plus $0.1 \%$ Triton X-100 and incubated in the blocking solution containing 5\% BSA plus $1 \times$ PBS plus $0.1 \%$ Triton X-100 for 2-3 hours. Subsequently, the slides were incubated in the primary antibody overnight, followed by 3 washes in PBS plus $0.1 \%$ Triton X-100 for 10 minutes each. After 2 hours of secondary antibody incubation, slides were washed 3 times in PBS plus $0.1 \%$ Triton X-100 for 10 minutes each. The specimens were overlaid with ProLong-Gold Antifade solution and a coverslip. All primary antibodies (Supplemental Table 5) were diluted 1:300 and secondary antibodies at 1:300. As a control, we used no primary as well as PRDM6 KO mice tissues. Otherwise, only validated and previously published antibodies were used. Fluorescence images were obtained by a Zeiss 4 laser confocal microscope or Leica SP8 confocal microscope, and the intensity was measured using the same laser output, gain, and offset for each set of antibodies tested. Images were quantified with ImageJ (NIH) and adjusted for the area.

Whole-mount staining. Whole-mount microscopy was carried out as previously described (47). Briefly, E9.5 mice were harvested and kept in ice-cold PBS for 5 minutes to drain blood from the umbilical cord and then fixed in $4 \% \mathrm{PFA}$ at $4^{\circ} \mathrm{C}$ and washed at room temperature. Samples were dehydrated in methanol/PBS series $(20 \%, 40 \%, 60 \%, 80 \%)$ for 30 minutes at room temperature and then $100 \%$ for the entire day. Samples were chilled over ice and bleached in $5 \% \mathrm{H}_{2} \mathrm{O}_{2}$ in methanol overnight at $4^{\circ} \mathrm{C}$, washed with $80 \%$ methanol/PBS for 
A
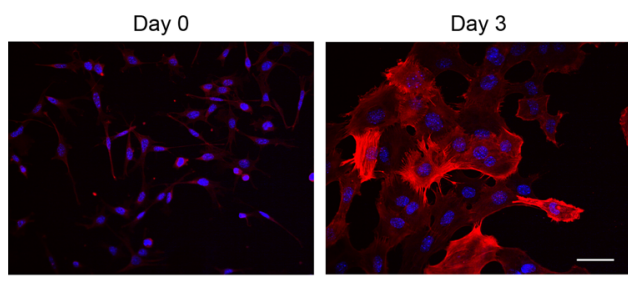

DAPI/aSMA

\section{B}

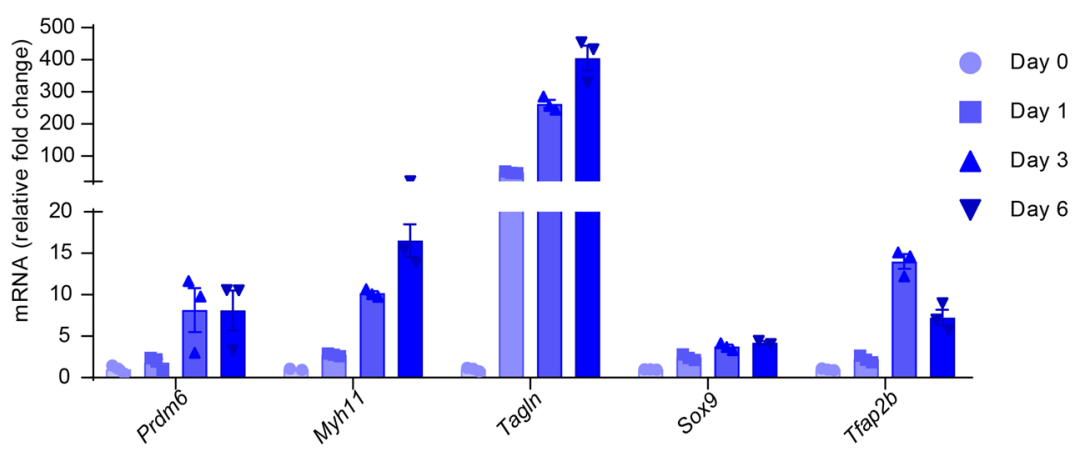

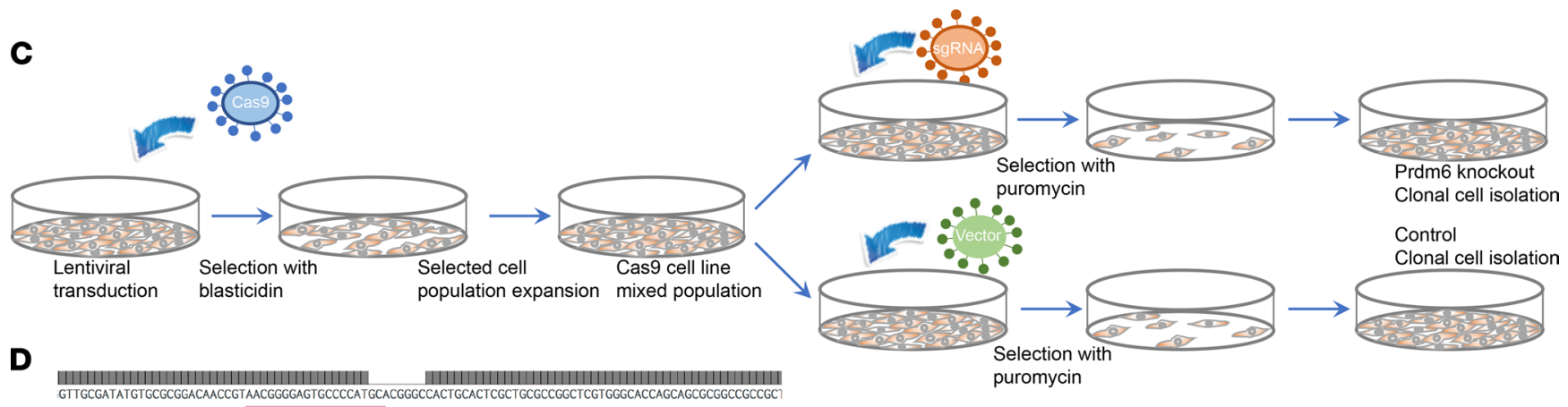

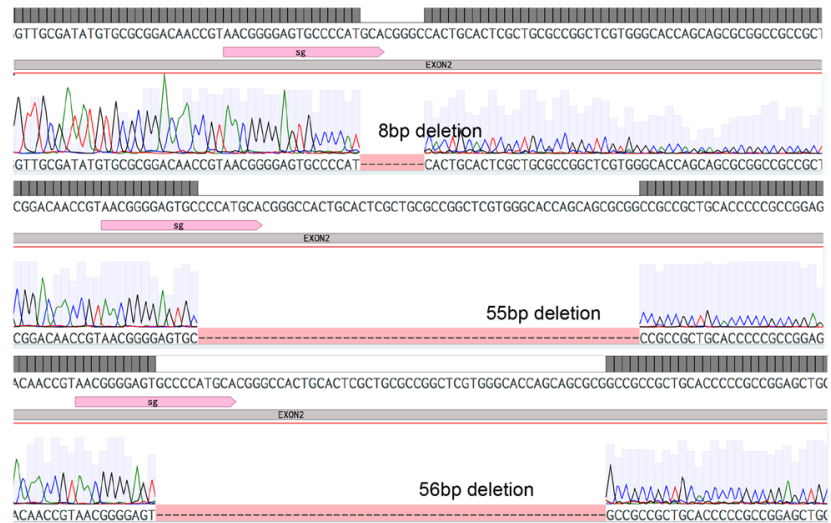

G

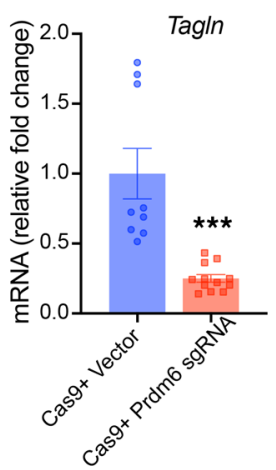

H

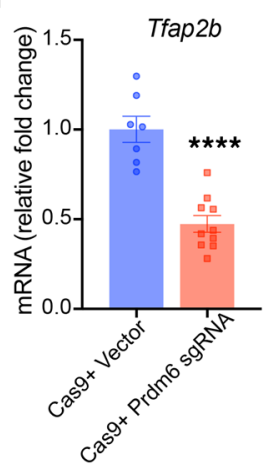

E

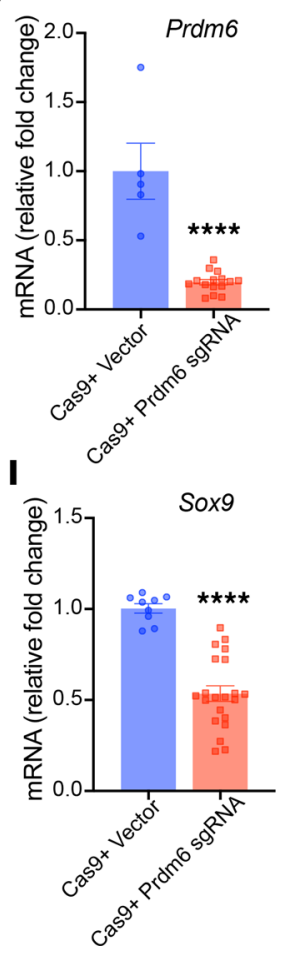

F
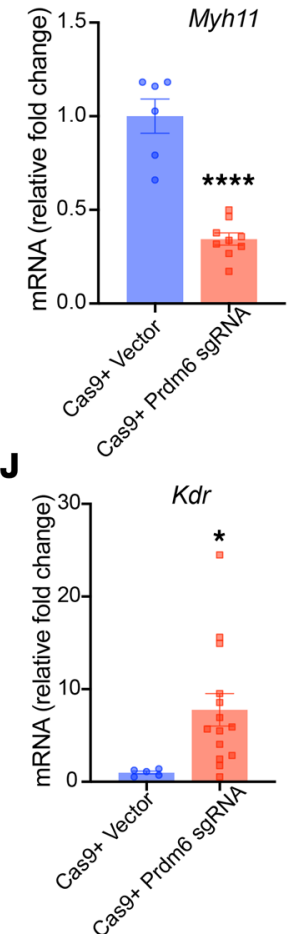

Figure 9. Characterization of Prdm6 in mouse NCC line. (A) Confocal images of 09-1 NCC line differentiated into smooth muscle cells after transfer to chemically defined medium at day 0 and day 3 and stained for $\alpha$ SMA (red) and DAPI (blue); scale bar: $50 \mu \mathrm{m}$. (B) mRNA level of specified genes in 09-1 cells at days $0,1,3$, and 6 of differentiation relative to Gapdh, quantified by qRT-PCR. (C) Schematic of screening of 09-1 transduced with Cas9 lentiviral vectors. Cell expressing Cas 9 were selected with blasticidin, transduced with sgRNA lentiviral vectors, and selected with puromycin to establish clonal cell lines. (D) Images of Sanger sequencing of segments of Prdm6 genes from 3 clonal cell lines with $8 \mathrm{bp}$, 55bp, or $56 \mathrm{bp}$ deletions. All 3 clonal cell lines were used in the experiment. qRT-PCR measurements of Prmd6 (E), Myh11 (F), Tagln (G), Tfap2b (H), Sox9 (I), and Kdr (J) mRNA expression in control and Cas9 + Prdm6 sgRNA cell lines. Each dot represents a biological replicate. Data are mean \pm SEM. For statistical analysis, groups were compared using unpaired 2-tailed $t$ test. ${ }^{*} P<0.05,{ }^{* *} P<0.001,{ }^{*}{ }^{* *} P<0.0001$. 
A

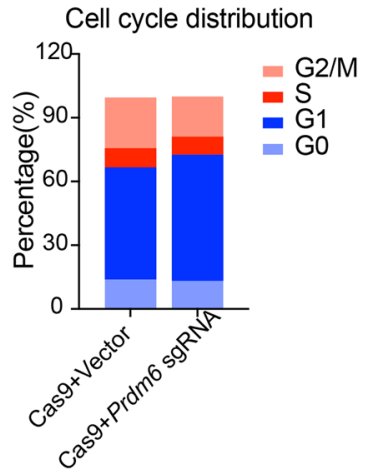

D

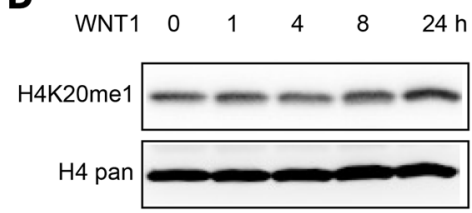

E

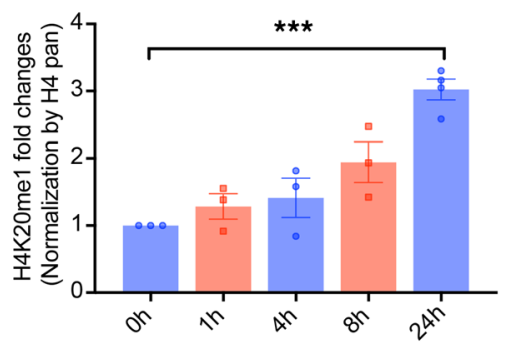

G

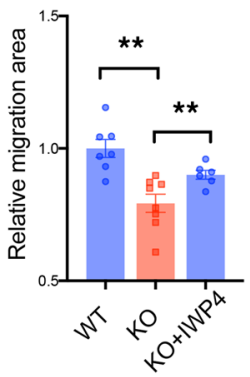

B

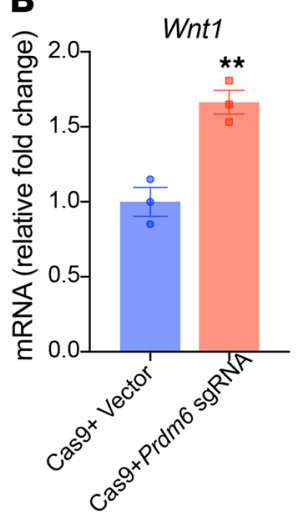

F Cas9+Vector DMSO
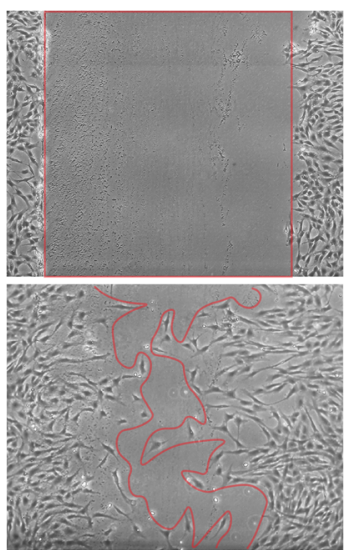

H
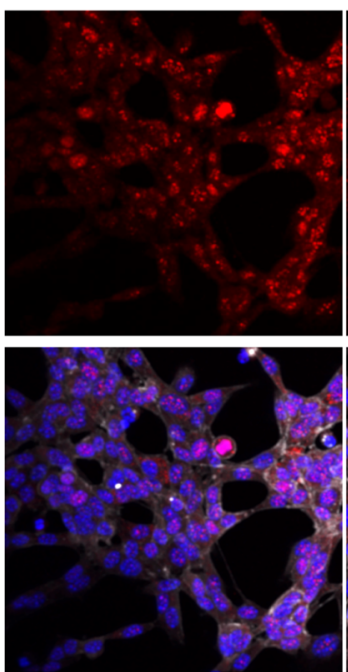

C

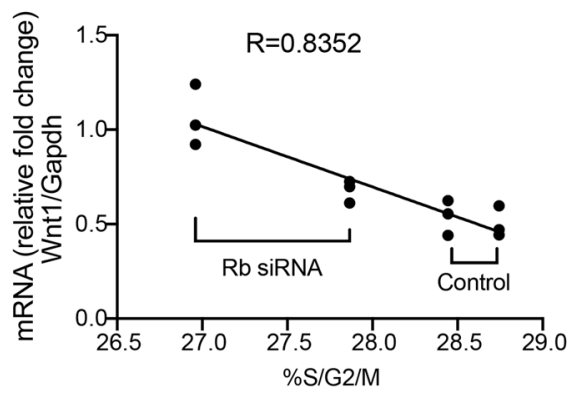

Cas9+Prdm6 sgRNA
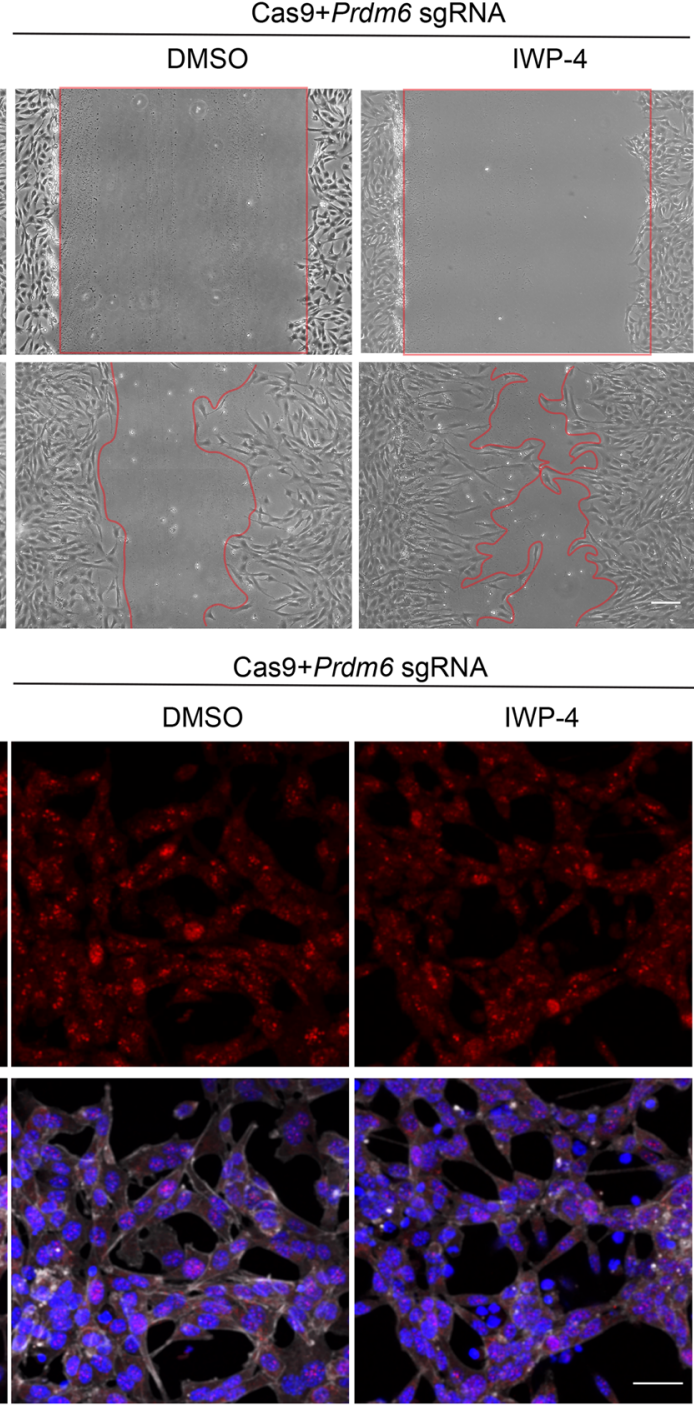

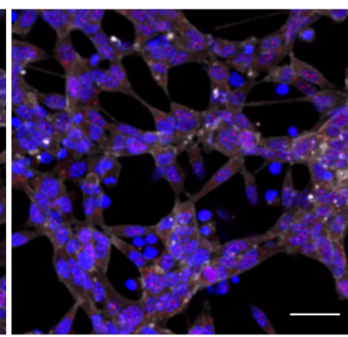

DAPI/H4K20me1/Phalloidin

Figure 10. The association between loss of Prdm6, elevated Wnt1, and reduced H4K20me1 in a mouse NCC line. (A) Images of light scattering by flow cytometry FACS demonstrating the cell cycle of control and Prdm6-KO 09-1 cells. (B) Wnt1 mRNA expression levels measured by qRT-PCR in WT and Prdm6 CRISPR KO 09-1 cells. (C) The mRNA expression levels of Wht1 and its relationship to percentage of cells in S/G2/M phase in 09-1 cells either treated with $R b$-siRNA or control siRNA. (D and E) H4K20me1 level of cells treated with PBS and WNT1 assayed by Western blot and its quantification (E). (F) Migration of Prdm6-deficient 09-1 cells in response to Wnt inhibitor IWP-4 compared with DMSO by wound assay and their quantification (C). Scale bar: $50 \mu \mathrm{m}$. (H) The confocal image of H4K2Ome1 in Prdm6-deficient 09-1 cells treated with Wnt inhibitor (IWP-4) or DMSO by staining DAPI (blue), phalloidin (gray), and antibodies against H4K2Ome1 (red). Scale bar: $50 \mu \mathrm{m}$. Each dot represents a biological replicate. The comparison between different groups was done by a 2-tailed unpaired $t$ test, and data are shown as mean \pm SEM. The comparisons between multiple groups were done by 1-way ANOVA. A Mann-Whitney test was conducted for non-normally distributed data ( $n=3-8$ per group). ${ }^{* *} P<0.001,{ }^{* * *} P<0.0001$. 
30 minutes at room temperature, and rehydrated with the methanol series in PBS $/ 0.2 \%$ Triton X-100 (fresh prepared): $80 \%, 60 \%, 40 \%, 20 \%, 0 \% ; 30$ minutes each at room temperature. For immunolabeling, pretreated samples were incubated in $1 \times \mathrm{PBS} / 0.2 \%$ Triton X-100/20\% DMSO/0.3 M glycine at $37^{\circ} \mathrm{C}$ for 24 hours and blocked in $1 \times \mathrm{PBS} / 0.2 \%$ Triton $\mathrm{X}-100 / 10 \% \mathrm{DMSO} / 6 \%$ goat serum at $37^{\circ} \mathrm{C}$ for 24 hours and subsequently washed with $1 \times \mathrm{PBS} / 0.2 \%$ Tween 20 with $10 \mu \mathrm{g} / \mathrm{mL}$ heparin and incubated with primary antibody in $1 \times$ $\mathrm{PBS} / 0.2 \%$ Tween 20 with $10 \mu \mathrm{g} / \mathrm{mL}$ heparin $/ 5 \% \mathrm{DMSO} / 3 \%$ donkey serum at $37^{\circ} \mathrm{C}$ for 24 hours. After several washes, samples were incubated with secondary antibody in $1 \times \mathrm{PBS} / 0.2 \%$ Tween 20 with $10 \mu \mathrm{g} / \mathrm{mL}$ heparin $/ 3 \%$ donkey serum at $37^{\circ} \mathrm{C}$ for 24 hours. For clearing, samples were incubated overnight in $50 \%, 80 \%$, and $100 \%$ tetrahydrofuran $/ \mathrm{H}_{2} \mathrm{O}$ (THF, Sigma-Aldrich, 186562-12X100ML) for 2 hours each time. Samples were then incubated in dichloromethane (DCM, Sigma-Aldrich, 270997-12X100ML) until they sunk to the bottom for 5 minutes and in dibenzyl ether (Sigma-Aldrich, 108014-1KG) until the samples were clear (20 minutes to 2 hours). We then examined samples using light sheet microscopy.

Isolation and culture of the neural tube. Isolation of the neural tube was carried out as described by Elise R. Pfaltzgraff, Nathan A. Mundell, and Patricia A. Labosky (48). For timed pregnancies, dams were checked in the morning for a vaginal plug and the embryo was considered as E0.5 at noon of the day the plug was observed. Mice were euthanized, and the embryos were removed at E9.5 and placed on ice-cold sterile DPBS. Using insulin needles, neural tubes were cut between the mid otic placode and the posterior edge of the fourth somite. The yolk sacs were kept for genotyping and the remaining embryonic tissues were discarded. The cardiac neural tube-containing segments were placed in collagenase/Dispase (Sigma-Aldrich, catalog 269-638) at room temperature for 8 minutes and subsequently washed in ice-cold sterile DPBS. The non-neural ectoderm was gently removed from the tissue, and the somites were separated away from the cardiac neural tube. The remaining parts of the mesoderm were gently removed using a cut-down $1 \mathrm{~mL}$ pipette tip. The isolated neural tube was washed for 30 seconds with culture medium (ES101-B) with fibroblast growth factor (GF003) and placed in the center of Matrigel-coated (Corning, catalog 354234) 24-well glass-bottom cell culture plate (Nest Scientific, catalog 801006) and incubated at $37^{\circ} \mathrm{C}$.

RNA extraction, $c D N A$ synthesis, and $q P C R$. The RNA was extracted using Qiagen miRNeasy Micro kit (catalog 1071023). Next, 100-500 ng of RNA was used for cDNA synthesis (iScript cDNA synthesis, BioRad, 1708890). No-RT and no-RNA controls were used to ensure DNase digestion and no residual contamination. The cDNA was diluted to different concentrations and eventually were diluted by 5 -fold to obtain qPCR values in the linear range. qPCR primers (Supplemental Table 6) were from PrimerBank. A dilution curve that included primers and qPCR Master Mix were utilized to ensure that PCR primers did not self-anneal and to reduce background amplification. The qPCR was performed with $2 \times$ SYBR Green Master Mix (Bio-Rad, 170-8880), 0.5 $\mu \mathrm{M}$ primer (forward and reverse), and $5 \mu \mathrm{L}$ of 1:5 diluted cDNA and rest water. All qPCR reactions had 3 technical replicates and at least 3 biological replicates per genotype. All values were normalized to the reference gene Gapdh.

Bulk RNA-Seq. The reads were trimmed for quality and length. All the reads were minimum base quality of Q30, and reads with minimum length of 45 were used for further analysis. The reads were aligned to the mouse UCSC reference genome $\operatorname{mm} 10(49,50)$ using TopHat2 $(51)$. The alignment data were converted to per-gene counts using Cufflinks (52) and further analyzed using Cuffdiff and R (http://www.r-project.org/index.html). The results were visualized using $\mathrm{R}$.

ScRNA-Seq. E9.5 embryos were harvested and briefly placed in ice-cold DPBS and then placed on a plastic petri dish resting on ice. Using insulin needles, the cardiac neural crest was dissected out from the mid otic placode to the posterior edge of the fourth somite. The tissues were removed and placed in a $1.5 \mathrm{~mL}$ conical snap-cap plastic tube on ice and $1 \times$ enzyme digestion buffer containing $100 \mu \mathrm{L}$ of Bacillus licheniformis stock (100 mg/mL, Sigma-Aldrich, P5380), $895 \mu \mathrm{L}$ of DPBS, and $5 \mu \mathrm{L}$ of $0.5 \mathrm{M}$ EDTA. The tube was quickly shaken thoroughly to mix and resuspend, incubated in ice-water bath, and triturated using a cut-down $1 \mathrm{~mL}$ pipette tip for 15 seconds every 2 minutes. Cells were filtered through a $40 \mu \mathrm{m}$ strainer, rinsed with $10 \mathrm{~mL}$ of ice-cold PBS/BSA, and weighed. Subsequently, $300 \mathrm{~g}$ of the cells were transferred to $15 \mathrm{~mL}$ conical tube, centrifuged at $300 \mathrm{~g}$ for 5 minutes at $4^{\circ} \mathrm{C}$, and the supernatant discarded. The pelleted cells were then resuspended in $100 \mu \mathrm{L}$ of ice-cold DPBS and counted on a Countess II (Thermo Fisher Scientific). Cell count and viability were determined using trypan blue dye exclusion.

QC. QC was evaluated from the distribution of QC covariates in data set visualizations and clustering after multiple times of analyzing the data and comparison with bulk RNA-Seq data. The analysis showed that use of fraction of counts from mitochondrial genes per barcode was too stringent and resulted in significant 
loss of signal since cardiovascular progenitor cells and migrating NCCs had a high degree of mitochondrial content. A more permissive QC threshold based on only 2 QC covariates, count depth and the number of genes per barcode, was considered as more appropriate. Capture sites that were either empty or contained multiple cells were filtered. DoubletFinder (53) and high-count depth threshold were also used to exclude doublets. Normalization protocol included count depth scaling (AKA CPM normalization). Gene normalization, which constitutes scaling gene counts to have zero mean and unit variance ( $z$ scores), was applied. Normalized data were log-transformed for use with downstream analysis. Data correction for biological effects based on cell cycle signals was avoided because of the presence of a large number of proliferating cells.

Single-cell sequencing pipeline. Ten thousand cells per sample were used for the 10x Genomics Cell Ranger pipeline with a combined human genome and the ZsGreen1 gene to obtain count matrices for the WT and KO conditions. There were an average of 2742 and 3102 counts per cell or an average of 1294 and 1432 unique genes detected in WT and KO conditions, respectively. Only samples with RIN greater than 6.5 were used. Library preparations and sequencing were performed by the Yale Center for Genome Analysis. Briefly, gel beads in emulsion (GEMs) were created using Chromium Next GEM Single Cell 3' Gel Beads v3.1 kit (10x Genomics) and barcoded. GEMs generated were used for cDNA synthesis and library preparation using the Chromium Single Cell 3' Library Kit v3.1 (10x Genomics) and sequenced on NovaSeq 6000 system using HiSeq 100 base pair reads and dual indexing.

ScRNA-Seq data alignment. scRNA-Seq samples were demultiplexed using MULTI-Seq and barcoded and pooled on droplet microfluidic emulsion and sequenced with the 10x Genomics Chromium system. Cell Ranger software v1.3.1 was used for library demultiplexing, FASTQ file generation, read alignment, and unique molecular identifier (UMI) quantification. The resulting raw UMI counts in each cell were normalized to their library size. The normalized counts were square-root transformed. We then used a combination of 3 metrics to find differentially expressed genes across conditions: (a) the binary logarithm of fold change between mean counts; (b) the Wasserstein or earth mover's distance (EMD); and (c) adjusted $P$ value from a 2-sided Mann-Whitney $U$ test with continuity and Benjamini-Hochberg correction, as described by Moon et al. (22). A $P$ value less than or equal to 0.01 was used as the threshold for statistical significance. The top 30 differentially expressed genes (up- or downregulated, ranked by Wasserstein distance, were represented in heatmaps. To identify putative cellular functions changed across conditions, we performed PANTHER-GO gene set enrichment analysis for each cluster (54) using the default human PANTHER-GO reference list as a background.

scRNA-Seq data analysis. We used the standard scRNA-Seq analysis pipeline for clustering (55). We removed cells with less than that 200 and more than 6000 raw UMIs to account for doublets. Next, we removed genes that were expressed in fewer than 3 cells and removed cells that expressed fewer than 200 genes. We also removed UMIs pertaining to mitochondrial and ribosomal genes. The counts in each cell were normalized to their library size. Then, normalized counts were square-root transformed, similar to a log transform but not requiring addition of a pseudo count. Data preprocessing was performed in Python (v3.7.4) using Scanpy (v1.4.6) $(56,57)$.

We visually observed batch effects between conditions in $2 \mathrm{D}$ cellular embeddings. To remove these batch effects for downstream analysis, we used an approximate batch-balanced k nearest neighbor $(\mathrm{kNN})$ graph for manifold learning (BB-kNN batch-effect correction) using Scanpy's fast approximation implementation $(56,57)$. For each cell, the 3 nearest neighboring cells in each condition were identified by Euclidean distance in 100-dimensional principal component analysis (PCA) space. This kNN graph was used as the basis for downstream analysis.

To visualize the scRNA-Seq data, we implemented 2 nonlinear dimension reduction methods and used the BB-kNN batch-corrected connectivity matrix as input for uniform manifold approximation and projection (UMAP) (58) and PHATE (22). UMAPs were generated using a minimum distance of 0.5. PHATE projections were generated with a gamma parameter of 0 .

For cell clustering, we used the Louvain community detection method (59) with the BB-kNN graph. We used high-resolution community detection and merged clusters based on expression of hand-picked markers for the cell types (Supplemental Figure 13). In order to minimize the effect of dropout for clustering, we imputed counts using Markov affinity-based graph imputation of cells (MAGIC) (60).

To find the differentially expressed genes between the WT and KO conditions in each cell type, we used 3 metrics: the 1-dimensional Wasserstein or EMD, an adjusted $P$ value from a 2-sided Mann-Whitney $U$ test with continuity and Benjamini-Hochberg correction, and the binary logarithm of fold-change between mean counts. Significance was set to an adjusted $P$ value of 0.01 . The EMD can be defined as 
the minimal cost to transform distribution to another and has been previously used to perform differential gene expression analysis (61-63).

O9-1 cell culture and differentiation and target gene KO in 09-1 cells. O9-1 cells were obtained from MilliporeSigma. Low-passage O9-1 cells (SCC049) were maintained on Matrigel-coated dishes in complete embryonic stem cell medium with $15 \%$ fetal bovine serum and leukemia inhibitory factor (ES-101-B) supplemented with bFGF.

The O9-Cas9 cell line was generated by transducing the 09-1 cells with lentivirus, which packaged an EFS-Cas9-T2A-blasticidin expression cassette; polybrene was used for improving virus infection efficiency. One day after lentiviral transduction, $10 \mu \mathrm{g} / \mathrm{mL}$ blasticidin was used for selection for 5 days to get a pure O9-Cas9 cell line. SgRNAs were designed (AACGGGGAGTGCCCCATGCA) and cloned into a backbone plasmid, U6-sgRNA-EFS-puromycin, and then packaged into lentivirus. The puromycin selection $(10 \mu \mathrm{g} / \mathrm{mL})$ was performed after lentiviral transduction for 3 days. The T7E1 assay was performed at day 7 after sgRNA lentiviral transduction to evaluate target gene KO efficiency.

Cell cycle distribution (Hoechst/pyronin Y). Single-cell suspensions were prepared and adjusted to contain $2 \times 10^{6}$ cells $/ \mathrm{mL}$. Single-cell suspensions were stained with Hoechst $(10 \mu \mathrm{g} / \mathrm{mL})$ and pyronin Y for 15 minutes at $37^{\circ} \mathrm{C}$.

Statistics. In vivo studies included a minimum of 3 mice in each group. In vitro studies were carried out in more than 3 independent experiments. The comparison between different groups was done by a 2 -tailed unpaired $t$ test. The normality was tested by Kolmogorov-Smirnov test. An $F$ statistic was calculated to determine whether variances were different between samples, and the $P$ values were then corrected with Welch's 2-tailed $t$ test. The comparisons between multiple groups were done by 1-way ANOVA. A Mann-Whitney test was conducted for non-normally distributed data. Fisher's exact test was carried out for the continuous variables. The preparation of graphs and all statistical analyses, including 2-tailed Student's $t$ tests, 2-way ANOVA (SigmaPlot), and testing for equal variance, were carried out using GraphPad Prism 8.1 Project software. $P$ values less than 0.05 were considered significant. Data are presented as mean \pm SEM. Fluorescence images were evaluated using ImageJ (NIH).

Code and data availability. All differential gene expression analyses from bulk and scRNA-Seq and their associated metrics have been deposited in NCBI's Gene Expression Omnibus (GSE195590).

Study approval. Animal procedures were conducted according to an approved protocol by the Yale University IACUC.

\section{Author contributions}

LH designed and conducted experiments, acquired data, analyzed data, and helped with preparing the manuscript. NL generated floxed mice. VG analyzed scRNA-Seq data. SM analyzed bulk RNA-Seq data. LY, YW, and JL analyzed data. AG and JR provided the mice. KKH and AE supervised the data analysis. $\mathrm{CH}$ helped with the writing of the manuscript and provided instruction on the preparation of the data. DVD supervised the analysis of scRNA-Seq data. AM designed and supervised the research studies and wrote the manuscript.

\section{Acknowledgments}

This work was supported by grants from NIH (5R35HL135767A to AM). The authors acknowledge the use of the shared imaging resources from the Yale Translational Research Imaging Center (Y-TRIC).

Address correspondence to: Arya Mani, Yale Cardiovascular Research Center, 300 George Street, New Haven, Connecticut, 06511, USA. Email: arya.mani@yale.edu.

1. Le Douarin NM, et al. Neural crest cell plasticity and its limits. Development. 2004;131(19):4637-4650.

2. Ruhrberg C, Schwarz Q. In the beginning: generating NCC diversity. Cell Adh Migr. 2010;4(4):622-630

3. Porras D, Brown CB. Temporal-spatial ablation of neural crest in the mouse results in cardiovascular defects. Dev Dyn. 2008;237(1):153-162.

4. Keyte A, Hutson MR. The neural crest in cardiac congenital anomalies. Differentiation. 2012;84(1):25-40.

5. Soldatov R, et al. Spatiotemporal structure of cell fate decisions in murine neural crest. Science. 2019;364(6444):eaas9536.

6. Tang W, Bronner ME. Neural crest lineage analysis: from past to future trajectory. Development. 2020;147(20):dev193193.

7. Tadic M, et al. Influence of white-coat hypertension on left ventricular deformation 2- and 3-dimensional speckle tracking study. Hypertension. 2016;67(3):592-596. 
8. Gewies A, et al. Prdm6 is essential for cardiovascular development in vivo. PLoS One. 2013;8(11):e81833.

9. Li N, et al. Mutations in the histone modifier PRDM6 are associated with isolated nonsyndromic patent ductus arteriosus. Am J Hum Genet. 2016;99(4):1000.

10. Nishibatake M, et al. Pathogenesis of persistent truncus arteriosus and dextroposed aorta in the chick embryo after neural crest ablation. Circulation. 1987;75(1):255-264.

11. Jiang X, et al. Fate of the mammalian cardiac neural crest. Development. 2000;127(8):1607-1616.

12. Zhao F, et al. Novel TFAP2B mutations that cause Char syndrome provide a genotype-phenotype correlation. Am J Hum Genet. 2001;69(4):695-703.

13. Mani A, et al. Syndromic patent ductus arteriosus: evidence for haploinsufficient TFAP2B mutations and identification of a linked sleep disorder. Proc Natl Acad Sci U S A. 2005;102(8):2975-2979.

14. Jiang XB, et al. Fate of the mammalian cardiac neural crest. Development. 2000;127(8):1607-1616.

15. George RM, et al. The heart of the neural crest: cardiac neural crest cells in development and regeneration. Development. 2020;147(20):dev188706.

16. Bergwerff $\mathrm{M}$, et al. Neural crest cell contribution to the developing circulatory system: implications for vascular morphology? Circ Res. 1998;82(2):221-231.

17. Kim HS, et al. Apoptosis and regulation of Bax and Bcl-X proteins during human neonatal vascular remodeling. Arterioscler Thromb Vasc Biol. 2000;20(4):957-963.

18. Tananari Y, et al. Role of apoptosis in the closure of neonatal ductus arteriosus. Jpn Circ J. 2000;64(9):684-688

19. Slomp J, et al. Differentiation, dedifferentiation, and apoptosis of smooth muscle cells during the development of the human ductus arteriosus. Arterioscler Thromb Vasc Biol. 1997;17(5):1003-1009.

20. Yajima I, et al. A subpopulation of smooth muscle cells, derived from melanocyte-competent precursors, prevents patent ductus arteriosus. PLoS One. 2013;8(1):e53183.

21. Plein A, et al. Neural crest cells in cardiovascular development. Curr Top Dev Biol. 2015;111:183-200.

22. Moon KR, et al. Visualizing structure and transitions in high-dimensional biological data. Nat Biol. 2019;37(12):1482-1492.

23. Boudreau N, et al. Fibronectin, hyaluronan, and a hyaluronan binding protein contribute to increased ductus arteriosus smooth muscle cell migration. Dev Biol. 1991;143(2):235-247.

24. Kleber M, et al. Neural crest stem cell maintenance by combinatorial Wnt and BMP signaling. J Cell Biol. 2005;169(2):309-320.

25. Wu Y, et al. PRDM6 is enriched in vascular precursors during development and inhibits endothelial cell proliferation, survival, and differentiation. J Mol Cell Cardiol. 2008;44(1):47-58.

26. Wu Y, et al. Suv4-20h1 promotes G1 to S phase transition by downregulating $\mathrm{p} 21^{\mathrm{WAF} 1 / \mathrm{CIP} 1}$ expression in chronic myeloid leukemia K562 cells. Oncol Lett. 2018;15(5):6123-6130.

27. Burstyn-Cohen T, Kalcheim C. Association between the cell cycle and neural crest delamination through specific regulation of G1/S transition. Dev Cell. 2002;3(3):383-395

28. Yang F, et al. SET8 promotes epithelial-mesenchymal transition and confers TWIST dual transcriptional activities. EMBO J. 2012;31(1):110-123.

29. Gonzalo S, et al. Role of the RB1 family in stabilizing histone methylation at constitutive heterochromatin. Nat Cell Biol. 2005;7(4):420-428.

30. Nguyen M, et al. The prostaglandin receptor EP4 triggers remodelling of the cardiovascular system at birth. Nature. 1997;390(6655):78-81.

31. Yu Y, et al. Genetic model of selective COX2 inhibition reveals novel heterodimer signaling. Nat Med. 2006;12(6):699-704

32. Huang $\mathrm{J}$, et al. Myocardin regulates expression of contractile genes in smooth muscle cells and is required for closure of the ductus arteriosus in mice. $J$ Clin Invest. 2008;118(2):515-525.

33. $\mathrm{Ng} \mathrm{SB}$, et al. Exome sequencing identifies MLL2 mutations as a cause of Kabuki syndrome. Nat Genet. 2010;42(9):790-793

34. Kleefstra T, et a1. Loss-of-function mutations in euchromatin histone methyl transferase 1 (EHMT1) cause the 9q34 subtelomeric deletion syndrome. Am J Hum Genet. 2006;79(2):370-377.

35. Yan S, et al. Epigenetic regulation of cardiac neural crest cells. Front Cell Dev Biol. 2021;9:678954

36. Ille F, et al. Wnt/BMP signal integration regulates the balance between proliferation and differentiation of neuroepithelial cells in the dorsal spinal cord. Dev Biol. 2007;304(1):394-408.

37. Martik ML, Bronner ME. Regulatory logic underlying diversification of the neural crest. Trends Genet. 2017;33(10):715-727.

38. Lee J, Zhou P. SETting the clock for histone H4 monomethylation. Mol Cell. 2010;40(3):345-346.

39. Oda $\mathrm{H}$, et al. Monomethylation of histone H4-lysine 20 is involved in chromosome structure and stability and is essential for mouse development. Mol Cell Biol. 2009;29(8):2278-2295.

40. Isaac CE, et al. The retinoblastoma protein regulates pericentric heterochromatin. Mol Cell Biol. 2006;26(9):3659-3671.

41. Bromberg KD, et al. The SUV4-20 inhibitor A-196 verifies a role for epigenetics in genomic integrity. Nat Chem Biol. 2017;13(3):317-324.

42. Burstyn-Cohen T, et al. Canonical Wnt activity regulates trunk neural crest delamination linking BMP/noggin signaling with G1/S transition. Development. 2004;131(21):5327-5339.

43. Laufer BI, et al. Associative DNA methylation changes in children with prenatal alcohol exposure. Epigenomics. 2015;7(8):1259-1274.

44. Chater-Diehl EJ, et al. Alteration of gene expression, DNA methylation, and histone methylation in free radical scavenging networks in adult mouse hippocampus following fetal alcohol exposure. PLoS One. 2016;11(5):e0154836.

45. Godino A, et al. Epigenetic landscape of amphetamine and methamphetamine addiction in rodents. Epigenetics. 2015;10(7):574-580.

46. Smith AK, et al. Prenatal antiepileptic exposure associates with neonatal DNA methylation differences. Epigenetics. 2012;7(5):458-463.

47. Yokomizo T, et al. Whole-mount three-dimensional imaging of internally localized immunostained cells within mouse embryos. Nat Protoc. 2012;7(3):421-431.

48. Pfaltzgraff ER, et al. Isolation and culture of neural crest cells from embryonic murine neural tube. J Vis Exp. 2012;(64):e4134

49. Kent WJ, et al. The human genome browser at UCSC. Genome Res. 2002;12(6):996-1006.

50. Frankish A, et al. GENCODE reference annotation for the human and mouse genomes. Nucleic Acids Res. 2019;47(d1):D766-D773. 
51. Kim D, et al. TopHat2: accurate alignment of transcriptomes in the presence of insertions, deletions and gene fusions. Genome Biol. 2013;14(4):R36.

52. Trapnell C, et al. Differential gene and transcript expression analysis of RNA-seq experiments with TopHat and Cufflinks. Nat Protoc. 2012;7(3):562-578.

53. McGinnis CS, et al. DoubletFinder: doublet detection in single-cell RNA sequencing data using artificial nearest neighbors. Cell Syst. 2019;8(4):329-337

54. Reimand J, et al. Pathway enrichment analysis and visualization of omics data using g:Profiler, GSEA, Cytoscape and EnrichmentMap. Nat Protoc. 2019;14(2):482-517.

55. Satija R, et al. Spatial reconstruction of single-cell gene expression data. Nat Biotechnol. 2015;33(5):495-502

56. Wolf FA, et al. SCANPY: large-scale single-cell gene expression data analysis. Genome Biol. 2018;19(1):15.

57. Polanski K, et al. BBKNN: fast batch alignment of single cell transcriptomes. Bioinformatics. 2020;36(3):964-965.

58. McInnes L, et al. Uniform Manifold Approximation and Projection for Dimension Reduction. https://arxiv.org/ pdf/1802.03426.pdf. Accessed January 24, 2022.

59. Blondel VD, et al. Fast unfolding of communities in large networks. J Stat Mech. 2008;10:10008.

60. van Dijk D, et al. Recovering gene interactions from single-cell data using data diffusion. Cell. 2018;174(3):716-729

61. Wang T, Nabavi S. SigEMD: a powerful method for differential gene expression analysis in single-cell RNA sequencing data. Methods. 2018;145:25-32.

62. Orlova DY, et al. Earth mover's distance (EMD): a true metric for comparing biomarker expression levels in cell populations PLoS One. 2016;11(3):e151859.

63. Ravindra NG, et al. Single-cell longitudinal analysis of SARS-CoV-2 infection in human airway epithelium identifies target cells, alterations in gene expression, and cell state changes. PLoS Biol. 2021;19(3):e3001143. 